\title{
SPACES OF COINVARIANTS AND FUSION PRODUCT I. FROM EQUIVALENCE THEOREM TO KOSTKA POLYNOMIALS
}

\author{
B. FEIGIN, M. JIMBO, R. KEDEM, S. LOKTEV, T. MIWA
}

\begin{abstract}
The fusion rule gives the dimensions of spaces of conformal blocks in the WZW theory. We prove a dimension formula similar to the fusion rule for spaces of coinvariants of affine Lie algebras $\widehat{\mathfrak{g}}$. An equivalence of filtered spaces is established between spaces of coinvariants of two objects: highest weight $\widehat{\mathfrak{g}}$-modules and tensor products of finite-dimensional evaluation representations of $\mathfrak{g} \otimes \mathbb{C}[t]$.

In the $\widehat{\mathfrak{s l}}_{2}$ case we prove that their associated graded spaces are isomorphic to the spaces of coinvariants of fusion products, and that their Hilbert polynomials are the level-restricted Kostka polynomials.
\end{abstract}

\section{INTRODUCTION}

First recall the notion of conformal blocks in the Wess-Zumino-Witten conformal field theory. Let $\mathfrak{g}$ be a simple Lie algebra over $\mathbb{C}$, let $\tilde{\mathfrak{g}}=\mathfrak{g} \otimes$ $\mathbb{C}\left[t, t^{-1}\right]$ be the loop algebra, and $\tilde{\mathfrak{g}}^{\{0\}}$ its completion with respect to power series in $t$. As a geometric part of the data consider a compact complex curve $C$ with chosen distinct points $p_{0}, \ldots, p_{N}$. We take these points to be regular, and choose local coordinates $t_{0}, \ldots, t_{N}$. By $\mathfrak{g}^{C \backslash\left\{p_{0}, \ldots, p_{N}\right\}}$ denote the Lie algebra of $\mathfrak{g}$-valued meromorphic functions on $C$ with possible poles at $p_{i}$. Local coordinates provide us with the inclusion

$$
\mathfrak{g}^{C \backslash\left\{p_{0}, \ldots, p_{N}\right\}} \rightarrow \tilde{\mathfrak{g}}^{\{0\}} \oplus \cdots \oplus \tilde{\mathfrak{g}}^{\{0\}} \oplus \mathbb{C} K
$$

sending a function into its Laurent expansion at each point. Here $\mathbb{C} K$ is the one-dimensional center. The central extension in the right hand side is given by the sum of canonical cocycles of each summand $\tilde{\mathfrak{g}}^{\{0\}}$. It is trivial on the image of $\mathfrak{g}^{C \backslash\left\{p_{0}, \ldots, p_{N}\right\}}$.

Let $L_{0}, \ldots, L_{N}$ be irreducible highest weight representations of a fixed level $k$ of $\widehat{\mathfrak{g}}^{\{0\}}=\tilde{\mathfrak{g}}^{\{0\}} \oplus \mathbb{C} K \oplus \mathbb{C} d$. Let $v_{i}$ be their highest weight vectors. I

Define the space of conformal blocks by

$$
\left\langle L_{0}, \ldots, L_{N}\right\rangle_{p_{0}, \ldots, p_{N}}^{C}=L_{0} \otimes \cdots \otimes L_{N} / \mathfrak{g}^{C \backslash\left\{p_{0}, \ldots, p_{N}\right\}}\left(L_{0} \otimes \cdots \otimes L_{N}\right) .
$$

Date: November 3, 2018.

${ }^{1}$ In the main text, we will consider reducible highest weight representations with cyclic vectors $v_{i}$. In this introduction, we restrict to irreducible representations in order to ease the presentation. 
In this paper, we call it the space of conformal coinvariants. Here we use the symbol $\otimes$ in place of the tensor product symbol $\otimes$ in order to emphasize that we consider the action of the Lie algebra (1.1).

If $L_{i}$ are integrable, then it was proved by [TUY] that these spaces are finite dimensional, and that their dimensions are given by the fusion rule, in particular, it depends only on representations and the genus of the curve (see Theorem 2.7 for the fusion rule when the curve is $\mathbb{C} P^{1}$ ).

Introduce a filtration on the space of conformal coinvariants in the following way. Let $v_{1}, \ldots, v_{N}$ be the highest weight vectors of representations $L_{1}, \ldots, L_{N}$, respectively. Then we have a map from $L_{0}$ to the space of conformal coinvariants given by

$$
L_{0} \simeq L_{0} \otimes v_{1} \otimes \cdots \otimes v_{N} \rightarrow\left\langle L_{0}, \ldots, L_{N}\right\rangle_{p_{0}, \ldots, p_{N}}^{C} .
$$

By a standard argument (see Subsection 2.4) one can show that the map (1.3) is surjective. This gives us a realization of the space of conformal coinvariants as a quotient of $L_{0}$. Since the representation $L_{0}$ is graded by degree of $t_{0}^{-1}$, our space inherits the corresponding increasing filtration.

In this paper, we study this filtration for rational $C$ and irreducible highest weight representations $L_{0}, \ldots, L_{N}$ of level $k \in \mathbb{Z}_{>0}$.

From this point we restrict to the case of rational $C$. We take $p_{0}=\infty$, and $p_{i}=z_{i} \in \mathbb{C}(1 \leq i \leq N)$. We use the local coordinates $t_{0}=1 / t$ and $t_{i}=t-z_{i}$. We take $L_{0}$ to be the level $k$ irreducible highest weight module whose degree zero subspace is the irreducible $\mathfrak{g}$-module $\pi_{\lambda}$ with highest weight $\lambda$. We denote this $L_{0}$ specifically by $L_{\lambda}^{(k)}(\infty)$. Similarly, we take highest weights $\lambda_{i}$ for $L_{i}(1 \leq i \leq N)$.

The space $L_{\lambda}^{(k)}(\infty)$ is generated from the highest weight vector $v_{0}$ by the action of $\mathfrak{g}[t]=\mathfrak{g} \otimes \mathbb{C}[t]$. Consider the mapping

$$
\Delta_{z}=\left(\phi_{z_{1}}^{-1} \otimes \cdots \otimes \phi_{z_{N}}^{-1}\right) \circ \Delta^{(N-1)}: U(\mathfrak{g}[t]) \rightarrow U(\mathfrak{g}[t]) \otimes \cdots \otimes U(\mathfrak{g}[t]),
$$

where $\phi_{z}$ is the automorphism of $U(\mathfrak{g}[t])$ changing $t$ to $t-z$. Here $z$ denotes the set of points $\left\{z_{1}, \ldots, z_{N}\right\}$.

For $x \in \mathfrak{g}[t]$ and $v^{(i)} \in L_{i}$, we have

$$
\left(x v^{(0)}\right) \otimes v^{(1)} \otimes \cdots \otimes v^{(N)}=v^{(0)} \otimes \Delta_{Z}(S(x))\left(v^{(1)} \otimes \cdots \otimes v^{(N)}\right)
$$

in the quotient space (1.2). Here $S$ is the antipode.

Let $A \subset U(\mathfrak{g}[t]) \otimes \cdots \otimes U(\mathfrak{g}[t])$ be the left ideal which annihilates $v_{1} \otimes$ $\cdots \otimes v_{N}$. The kernel of the map (1.3) contains $\Delta_{z}^{-1}(S(A)) L_{\lambda}^{(k)}(\infty)$. In fact, we will show that they are equal (see Proposition 2.19).

In order to prove this statement, we introduce the notion of fusion right ideal $X_{1} \circledast \ldots \circledast X_{N}(z)=\Delta_{z}^{-1}(B)$ for right ideals $X_{1}, \ldots, X_{N} \subset U(\mathfrak{g}[t])$ by replacing $S(A)$ with

$$
B=\sum_{i=1}^{N} U(\mathfrak{g}[t])^{\otimes(i-1)} \otimes X_{i} \otimes U(\mathfrak{g}[t])^{\otimes(N-i)} .
$$


It is known that the dimension of the space of conformal blocks is given in terms of the multiplication rule in the Verlinde algebra (see Section 2.3 for the definition of the Verlinde algebra). In Theorem 2.9, we give a similar multiplicative formula for the dimension of the space of coinvariants

$$
L_{\lambda}^{(k)}(\infty) /\left(X_{1} \circledast \ldots \circledast X_{N}(Z)\right) L_{\lambda}^{(k)}(\infty)
$$

under a certain condition on the right ideals $X_{i}$. In particular, this gives a proof of the equality of the kernel of $(1.3)$ with the subspace $\left(X_{1} \circledast \ldots \circledast\right.$ $\left.X_{N}(\mathfrak{Z})\right) L_{\lambda}^{(k)}(\infty)$ by taking $X_{i}=S\left(\mathfrak{X}_{\lambda_{i}}^{\prime}\right)+B_{1}$, where the left ideal $\mathfrak{X}_{\lambda_{i}}^{\prime} \subset U(\mathfrak{g})$ is defined by $U(\mathfrak{g}) / \mathfrak{X}_{\lambda_{i}}^{\prime} \simeq \pi_{\lambda_{i}}$ and $B_{1}=(\mathfrak{g} \otimes t \mathbb{C}[t]) U(\mathfrak{g}[t])$. Therefore, the filtration of the conformal coinvariants (1.2) can be studied in the form (1.5) as the coinvariants with respect to the fusion right ideal.

Let us consider another version of this construction. By $\pi\left(L_{i}\right)$ denote the finite-dimensional representation of $\mathfrak{g}$ generated by the highest weight vector $v_{i}$. Then one can easily deduce from (1.3) that the following map is also surjective (see Subsection 2.4)

$$
v_{0} \otimes \pi\left(L_{1}\right) \otimes \cdots \otimes \pi\left(L_{N}\right) \rightarrow\left\langle L_{0}, \ldots, L_{N}\right\rangle_{p_{0}, \ldots, p_{N}}^{C} .
$$

Let $I_{\lambda}^{(k)} \subset \mathfrak{g} \otimes \mathbb{C}[t]$ be the left ideal which annihilates $v_{0}$. Then a similar argument shows that we have a surjective map

$$
\pi\left(L_{1}\right) \otimes \cdots \otimes \pi\left(L_{N}\right) / S\left(I_{\lambda}^{(k)}\right) \rightarrow\left\langle L_{0}, \ldots, L_{N}\right\rangle_{p_{0}, \ldots, p_{N}}^{C} .
$$

We will show also that these spaces are in fact isomorphic (see Example 1 in Subsection 3.2).

To describe the filtration on the space of conformal coinvariants in this setting, we use the notion of filtered tensor product introduced in FD. It is the tensor product of cyclic $\mathfrak{g}$-modules $V_{1}, \cdots, V_{N}$ considered as the evaluation $\mathfrak{g}[t]$-modules with distinct parameters $z_{i}$. Upon choosing cyclic vectors $v_{i} \in V_{i}$ we can define a $\mathfrak{g}$-equivariant filtration. Namely, the vector $v_{1} \otimes \cdots \otimes v_{N}$ is cyclic in the tensor product with respect to the action of $\mathfrak{g}[t]$, so the whole representation is a quotient of $U(\mathfrak{g}[t])$. We have a grading of $\mathfrak{g}[t]$ and therefore of $U(\mathfrak{g}[t])$ by the degree in $t$, so the quotient inherits the corresponding filtration.

In our case we should choose the highest vectors of $\pi\left(L_{i}\right)$ as cyclic to obtain the same filtration as given by (1.5). The associated graded space of the filtered tensor product $[\mathrm{FZ}$

$$
V_{1} * \cdots * V_{N}(Z)=\operatorname{gr} \mathcal{F}_{Z}\left(V_{1}, \cdots, V_{N}\right)
$$

is called in $\mathrm{FL}$ the fusion product.

For $\mathfrak{g}=\mathfrak{s l}_{2}$ we prove that the Hilbert polynomial of this graded space is independent of the choice of points and coincides with the level-restricted Kostka polynomial (for definition see [SS]). This calculation uses the fusion product. The result of $[\mathrm{FF}$ enables us to give an upper estimate of the Hilbert polynomial for the quotient of the filtered tensor product. The 
resulting formula coincides with the known fermionic formula for the levelrestricted Kostka polynomial. Since at $q=1$, the level-restricted Kostka polynomial gives the dimension of the conformal blocks, which is given by the fusion rule, we can conclude that the fermionic formula is exact.

The plan of the paper is as follows. In Section 2, we give the definition of the fusion right ideals in $U(\mathfrak{g}[t])$, and prove the fusion rule for the dimensions of the spaces of coinvariants of integrable highest weight $\widehat{\mathfrak{g}}$-modules. In Section 3, we establish an equivalence between spaces of coinvariants of irreducible highest weight $\widehat{\mathfrak{g}}$-modules and those of filtered tensor products of cyclic $\mathfrak{g}$-modules. We give a brief review on the Kostka polynomials, and propose the definition of fusion Kostka polynomials. In Section 4, we prove that in the case of $\mathfrak{g}=\widehat{\mathfrak{s l}}_{2}$, the associated graded space of the space of conformal coinvariants is independent of the choice of insertion points, and that its Hilbert polynomial is the level-restricted Kostka polynomial. In Appendix we discuss behavior of fusion ideals and spaces of coinvariants when points collide.

\section{Formula FOR THE DIMENSION}

In this section we introduce the notion of fusion right ideals and consider spaces of coinvariants with respect to them. We give a formula for the dimension of the latter in terms of the Verlinde algebra (see Theorem 2.9).

2.1. Fusion of ideals. Let $\mathfrak{g}$ be a complex Lie algebra. By $\mathfrak{g}[t]$ denote the Lie algebra $\mathfrak{g} \otimes \mathbb{C}[t]$. When there is no fear of confusion, we will identify $\mathfrak{g}$ with $\mathfrak{g} \otimes t^{0} \subset \mathfrak{g}[t]$. For $z \in \mathbb{C}$ we denote by $\phi_{z}$ the automorphism of $\mathfrak{g}[t]$ sending $x \otimes t^{n}$ into $x \otimes(t-z)^{n}(x \in \mathfrak{g})$.

Let $z=\left(z_{1}, \ldots, z_{N}\right)$ be a set of complex numbers. Throughout this paper we assume that

$$
z_{i} \neq z_{j} \quad(i \neq j)
$$

Consider the map $\mathfrak{g}[t] \rightarrow \oplus^{N} \mathfrak{g}[t]$ which sends $x \in \mathfrak{g}[t]$ to $\phi_{z_{1}}^{-1}(x) \oplus \cdots \oplus \phi_{z_{N}}^{-1}(x)$. By the universal property it induces the map $U(\mathfrak{g}[t]) \rightarrow U(\mathfrak{g}[t])^{\otimes N}$ given by

$$
\Delta_{z}=\left(\phi_{z_{1}} \otimes \cdots \otimes \phi_{z_{N}}\right)^{-1} \circ \Delta^{(N-1)},
$$

where the iterated coproduct is defined by $\Delta^{(n-1)}=(\Delta \otimes$ id $) \circ \Delta^{(n-2)}$, $\Delta^{(1)}=\Delta$.

Definition 1. Let $X_{1}, \ldots, X_{N}$ be right ideals in $U(\mathfrak{g}[t])$. Define the fusion right ideal by

$$
X_{1} \circledast \ldots \circledast X_{N}(z)=\left(\Delta_{z}\right)^{-1}\left(\sum_{i=1}^{N} U(\mathfrak{g}[t])^{\otimes(i-1)} \otimes X_{i} \otimes U(\mathfrak{g}[t])^{\otimes(N-i)}\right) .
$$

Throughout this paper, for simplicity of notation, we denote as

$$
I^{(N)}\left(X_{1}, \ldots, X_{N}\right)=\sum_{i=1}^{N} U(\mathfrak{g}[t])^{\otimes(i-1)} \otimes X_{i} \otimes U(\mathfrak{g}[t])^{\otimes(N-i)} .
$$


The following Proposition validates this definition.

Proposition 2.1. If the right ideals $X_{i}=\mathfrak{a}_{i} U(\mathfrak{g}[t])$ are generated by Lie subalgebras $\mathfrak{a}_{i} \subset U(\mathfrak{g}[t])$, then the fusion right ideal is given by

$$
X_{1} \circledast \ldots \circledast X_{N}(z)=\left(\bigcap_{i=1}^{N} \phi_{z_{i}}\left(\mathfrak{a}_{i}\right)\right) U(\mathfrak{g}[t]) .
$$

As an example, consider the two-sided ideal of $U(\mathfrak{g}[t])$ consisting of elements which have $M$-fold zeros at the origin,

$$
B_{M}=\left(\mathfrak{g} \otimes t^{M} \mathbb{C}[t]\right) U(\mathfrak{g}[t]) .
$$

Let

$$
B_{M, z}=\left(\mathfrak{g} \otimes\left(t-z_{1}\right)^{M} \ldots\left(t-z_{N}\right)^{M} \mathbb{C}[t]\right) U(\mathfrak{g}[t])
$$

be the two-sided ideal consisting of elements which have $M$-fold zeros at the points in $z$. Then we have

$$
B_{M} \circledast \ldots \circledast B_{M}(z)=B_{M, z} .
$$

For the proof of Proposition 2.1 we use the following fact, which is a corollary of the Poincaré-Birkhoff-Witt (PBW) theorem.

Lemma 2.2. Let $\mathfrak{a}$ be a Lie algebra, and let $\mathfrak{b} \subset \mathfrak{a}$ be its Lie subalgebra. Take a basis of $\mathfrak{b},\left\{B_{i}\right\}_{i \in I}$, and extend it to a basis of $\mathfrak{a}$ by adjoining $\left\{A_{j}\right\}_{j \in J}$. $W$ assume that the index set $I$ and $J$ are ordered. Then the set of elements in $U(\mathfrak{a})$

$$
B_{i_{1}} \cdots B_{i_{m}} A_{j_{1}} \cdots A_{j_{n}} \quad\left(i_{1} \geq \cdots \geq i_{m} ; j_{1} \geq \cdots \geq j_{n}\right)
$$

with $m \geq 1, n \geq 0$ forms a basis of $\mathfrak{b} U(\mathfrak{a})$, and the one with $m=0, n \geq 0$ forms a basis of $U(\mathfrak{a}) / \mathfrak{b} U(\mathfrak{a})$.

Lemma 2.3. Let $V_{i}$ be a vector space with countable basis, and let $W_{i}$ be a subspace of $V_{i}, i=1, \cdots, N$. Then the kernel of the map

$$
V_{1} \otimes \cdots \otimes V_{N} \rightarrow\left(V_{1} / W_{1}\right) \otimes \cdots \otimes\left(V_{N} / W_{N}\right)
$$

is equal to

$$
\sum_{i=1}^{N} V_{1} \otimes \cdots \otimes V_{i-1} \otimes W_{i} \otimes V_{i+1} \otimes \cdots \otimes V_{N} .
$$

Proof. Choosing a filtration

$$
V_{i}=\bigcup_{a=0}^{\infty} V_{i, a}, \quad V_{i, a} \subset V_{i+1, a}
$$

such that $\operatorname{dim} V_{i, a}$ is finite, one can reduce the proof to the case where all $V_{i}$ are finite-dimensional. Then the statement follows by dimension counting.

Proposition 2.1 is a consequence of the following fact. 
Proposition 2.4. Let $\mathfrak{a}$ be a Lie algebra with a countable basis, and let $\mathfrak{a}_{1}, \ldots, \mathfrak{a}_{N}$ be its Lie subalgebras. Then,

$$
\left(\Delta^{(N-1)}\right)^{-1}\left(\sum_{i=1}^{N} U^{\otimes(i-1)} \otimes \mathfrak{a}_{i} U \otimes U^{\otimes(N-i)}\right)=\left(\mathfrak{a}_{1} \cap \cdots \cap \mathfrak{a}_{N}\right) U,
$$

where $U=U(\mathfrak{a})$.

Proof. The inclusion $\supset$ is clear. Therefore, by using Lemma 2.3, it is enough to show that the mapping

$$
p: U /\left(\mathfrak{a}_{1} \cap \cdots \cap \mathfrak{a}_{N}\right) U \rightarrow\left(U / \mathfrak{a}_{1} U\right) \otimes \cdots \otimes\left(U / \mathfrak{a}_{N} U\right),
$$

which is induced from $\Delta^{(N-1)}$, is injective. We prove this statement by induction on $N$. The case $N=1$ is obvious. We prove the case $N=2$. Take a basis $\left\{A_{\alpha}\right\} \sqcup\left\{B_{\beta}\right\} \sqcup\left\{C_{\gamma}\right\} \sqcup\left\{D_{\delta}\right\}$ of $\mathfrak{a}$ such that $\left\{A_{a}\right\}$ is a basis of $\mathfrak{a}_{1} \cap \mathfrak{a}_{2},\left\{A_{a}\right\} \sqcup\left\{B_{b}\right\}$ is a basis of $\mathfrak{a}_{1}$, and $\left\{A_{a}\right\} \sqcup\left\{C_{c}\right\}$ is a basis of $\mathfrak{a}_{2}$. We fix an ordering of each set $\left\{A_{\alpha}\right\},\left\{B_{\beta}\right\},\left\{C_{\gamma}\right\},\left\{D_{\delta}\right\}$. Write $x \in U$ as a linear combination of the PBW basis using the ordering:

$$
x=\sum c_{\alpha_{1}, \ldots, \delta_{d}} A_{\alpha_{1}} \cdots A_{\alpha_{a}} B_{\beta_{1}} \cdots B_{\beta_{b}} C_{\gamma_{1}} \cdots C_{\gamma_{c}} D_{\delta_{1}} \cdots D_{\delta_{d}}
$$

Here and after, we do not bother to mention a necessary ordering for the indices.

Let

$$
\pi: U \otimes U \rightarrow\left(U / \mathfrak{a}_{1} U\right) \otimes\left(U / \mathfrak{a}_{2} U\right)
$$

be the canonical surjection. We want to show that if $\pi \circ \Delta(x)=0$ then $x$ belongs to $\left(\mathfrak{a}_{1} \cap \mathfrak{a}_{2}\right) U$.

Let $F^{l} U$ be the filtration of $U$ defined as follows.

$$
F^{0} U=\mathbb{C} \cdot 1, \quad F^{l+1} U=F^{l} U+\mathfrak{a} F^{l} U .
$$

One can define a filtration of $U \otimes U$ by setting

$$
F^{l}(U \otimes U)=\sum_{l_{1}+l_{2}=l} F^{l_{1}} U \otimes F^{l_{2}} U
$$

and further induce a filtration of $\left(U / \mathfrak{a}_{1} U\right) \otimes\left(U / \mathfrak{a}_{2} U\right)$. By Lemma 2.2, the elements of the form $C_{\gamma_{1}} \cdots C_{\gamma_{c}} D_{\delta_{i_{1}}} \cdots D_{\delta_{i_{d^{\prime}}}}$ (resp., $B_{\beta_{1}} \cdots B_{\beta_{b}} D_{\delta_{j_{1}}} \cdots D_{\delta_{j_{d^{\prime \prime}}}}$ ) form a basis of $U / \mathfrak{a}_{1} U$ (resp., $U / \mathfrak{a}_{2} U$ ). We have the basis of $\left(U / \mathfrak{a}_{1} U\right) \otimes$ $\left(U / \mathfrak{a}_{2} U\right)$ consisting of the tensor product of these bases. Moreover, those which satisfies $c+b+d^{\prime}+d^{\prime \prime}=l$, form a basis of $\left(F^{l} / F^{l-1}\right)\left(\left(U / \mathfrak{a}_{1} U\right) \otimes\right.$ $\left.\left(U / \mathfrak{a}_{2} U\right)\right)$. Let us denote them by $X_{\gamma_{1}, \ldots, \gamma_{c}, \delta_{i_{1}} \ldots, \delta_{d_{d^{\prime}}} ; \beta_{1}, \ldots, \beta_{b}, \delta_{j_{1}}, \ldots, \delta_{d^{\prime \prime}}}$.

Note that $\pi \circ \Delta(1) \neq 0$. Suppose that $x \in F^{l} U(l \geq 1)$ and $\pi \circ \Delta(x)=0$. We will show that $x \in\left(\mathfrak{a}_{1} \cap \mathfrak{a}_{2}\right) U+F^{l-1} U$. Write $x$ as (2.5). If a monomial in this expression is such that $a \geq 1$ then it belongs to $\left(\mathfrak{a}_{1} \cap \mathfrak{a}_{2}\right) U$ and the kernel of $\pi \circ \Delta$. Therefore, one can assume that only the terms with $a=0$ appear in (2.5).

We want to show that

$$
c_{\beta_{1}, \ldots, \delta_{d}}=0
$$


if $b+c+d=l$. Consider the $2^{l}$ monomials that are obtained by expanding the product $\Delta\left(B_{\beta_{1}}\right) \cdots \Delta\left(B_{\beta_{b}}\right) \Delta\left(C_{\gamma_{1}}\right) \cdots \Delta\left(C_{\gamma_{c}}\right) \Delta\left(D_{\delta_{1}}\right) \cdots \Delta\left(D_{\delta_{d}}\right)$. If a monomial is such that either one of $B_{\beta}$ is sent to the first component of the tensor product $U \otimes U$, or one of $C_{\gamma}$ to the second, then the image of that monomial by the surjection $\pi$ belongs to $F^{l-1}\left(\left(U / \mathfrak{a}_{1} U\right) \otimes\left(U / \mathfrak{a}_{2} U\right)\right)$. Therefore, $\pi \circ \Delta\left(B_{\beta_{1}} \cdots D_{\delta_{d}}\right) \in\left(F^{l} / F^{l-1}\right)\left(\left(U / \mathfrak{a}_{1} U\right) \otimes\left(U / \mathfrak{a}_{2} U\right)\right)$ is the sum of $2^{d}$ elements $X_{\gamma_{1}, \ldots, \gamma_{c}, \delta_{i_{1}} \ldots, \delta_{d^{\prime}} ; \beta_{1}, \ldots, \beta_{b}, \delta_{j_{1}}, \ldots, \delta_{d_{d^{\prime \prime}}}}$ where $\left\{i_{1} \ldots, i_{d^{\prime}}\right\} \sqcup\left\{j_{1} \ldots, j_{d^{\prime \prime}}\right\}$ is a partition of the index set $\{1, \ldots, d\}$. Let us denote this sum by $Y_{\beta_{1}, \ldots, \delta_{d}}$. The elements $Y_{\beta_{1}, \ldots, \delta_{d}}$ that are obtained from $x$ are linearly independent. Therefore, we have (2.6).

Finally, suppose that the statement is true for $N-1(N \geq 3)$. It means that the mapping

$$
U /\left(\mathfrak{a}_{1} \cap \cdots \cap \mathfrak{a}_{N-1}\right) U \rightarrow\left(U / \mathfrak{a}_{1} U\right) \otimes \cdots \otimes\left(U / \mathfrak{a}_{N-1} U\right)
$$

is injective. From this follows that

$$
p_{1}:\left(U /\left(\mathfrak{a}_{1} \cap \cdots \cap \mathfrak{a}_{N-1}\right) U\right) \otimes\left(U / \mathfrak{a}_{N} U\right) \rightarrow\left(U / \mathfrak{a}_{1} U\right) \otimes \cdots \otimes\left(U / \mathfrak{a}_{N} U\right)
$$

is also injective. Consider the mapping

$$
p_{2}: U /\left(\mathfrak{a}_{1} \cap \cdots \cap \mathfrak{a}_{N}\right) U \rightarrow\left(U /\left(\mathfrak{a}_{1} \cap \cdots \cap \mathfrak{a}_{N-1}\right) U\right) \otimes\left(U / \mathfrak{a}_{N} U\right)
$$

induced from $\Delta$. Since $\Delta^{(N-1)}=\left(\Delta^{(N-2)} \otimes \mathrm{id}\right) \circ \Delta$, we have $p=p_{1} \circ p_{2}$. Therefore, it is enough to show that $p_{2}$ is injective. This is nothing but the case $N=2$.

Note that $\mathfrak{g}[t]$ and therefore $U(\mathfrak{g}[t])$ are graded by the degree in $t$.

Definition 2. We call a right ideal $X \subset U(\mathfrak{g}[t])$ a congruence ideal if $X$ is graded and $X \supset B_{M}$ for a sufficiently large $M$.

Quite generally, let $V$ be a left module over a Lie algebra $\mathfrak{a}$. For a left ideal $X \subset U(\mathfrak{a})$, we denote by $V^{X}=\{v \in V \mid x v=0 \forall x \in X\}$ the space of $X$ invariants. Likewise, for a right ideal $Y \subset U(\mathfrak{a})$, we denote by $V / Y=V / Y V$ the space of $Y$-coinvariants. We have a canonical isomorphism

$$
(V / Y)^{*} \simeq\left(V^{*}\right)^{S(Y)}
$$

where $V^{*}=\operatorname{Hom}_{\mathbb{C}}(V, \mathbb{C})$ and $S: U(\mathfrak{a}) \rightarrow U(\mathfrak{a})$ stands for the antipode (the anti-automorphism such that $S(x)=-x$ for $x \in \mathfrak{a}$ ). We will consider coinvariant spaces with respect to fusion right ideals.

2.2. Affine Lie algebras. In this subsection we fix our notation concerning affine Lie algebras and integrable modules.

Let $\mathfrak{g}$ be a simple Lie algebra. Fix a triangular decomposition $\mathfrak{g}=$ $\mathfrak{n}_{+} \oplus \mathfrak{h} \oplus \mathfrak{n}_{-}$, where $\mathfrak{h} \subset \mathfrak{g}$ is the Cartan subalgebra and $\mathfrak{n}_{+}$(resp. $\mathfrak{n}_{-}$) is the nilpotent subalgebra of all positive (resp. negative) root vectors. The invariant bilinear form $(\mid)$ on $\mathfrak{g}$ is normalized as $(\theta \mid \theta)=2$, where $\theta$ is the maximal root. Let $P$ be the weight lattice and $P_{+}$be the set of dominant integral weights. For a non-negative integer $k \in \mathbb{Z}_{\geq 0}$, we set 
$P_{+}^{(k)}=\left\{\lambda \in P_{+} \mid\left\langle\lambda, \theta^{\vee}\right\rangle \leq k\right\}$, where $\theta^{\vee}$ denotes the maximal coroot. For $\lambda \in P_{+}$, we denote by $\pi_{\lambda}$ the irreducible representation with highest weight $\lambda$. We will write $\lambda^{*}=-w_{0}(\lambda)$ for the highest weight of the dual representation $\pi_{\lambda}^{*}, w_{0}$ being the longest element of the Weyl group $W$. If $\lambda \in P_{+}^{(k)}$, then $\lambda^{*} \in P_{+}^{(k)}$.

Let $\widehat{\mathfrak{g}}=\mathfrak{g} \otimes \mathbb{C}\left[t, t^{-1}\right] \oplus \mathbb{C} K \oplus \mathbb{C} d$ be the affine Lie algebra of non-twisted type. Here $K$ is a central element, $d=t d / d t$,

$$
\begin{gathered}
{[x \otimes f, y \otimes g]=[x, y] \otimes f g+\Omega_{0}(x \otimes f, y \otimes g) K} \\
\left(x, y \in \mathfrak{g}, f, g \in \mathbb{C}\left[t, t^{-1}\right]\right),
\end{gathered}
$$

and the canonical cocycle is given by

$$
\Omega_{0}(x \otimes f, y \otimes g)=(x \mid y) \operatorname{Res}_{t=0} d f \cdot g .
$$

Denote by $\Lambda_{i}$ the fundamental weights $(0 \leq i \leq r ; r=\operatorname{dim} \mathfrak{h})$ and by $\delta$ the null root. Let $\Lambda=\lambda+k \Lambda_{0}+a \delta(\lambda \in P, a \in \mathbb{C})$ be an affine weight of level $k$. For an element $w$ of the affine Weyl group $W_{\text {aff }}$, we set

$$
\begin{aligned}
& w \circ\left(\lambda+k \Lambda_{0}+a \delta\right)=w(\lambda, k)+k \Lambda_{0}+\left(a-d_{w}(\lambda, k)\right) \delta, \\
& w(\lambda, k) \in P, d_{w}(\lambda, k) \in \mathbb{Z},
\end{aligned}
$$

where $w \circ \Lambda=w(\Lambda+\rho)-\rho$ stands for the shifted action and $\rho=\sum_{i=0}^{r} \Lambda_{i}$.

We say a $\widehat{\mathfrak{g}}$-module has level $k$ if $K$ acts as $k$ times the identity on it. For $k \in \mathbb{Z}_{\geq 0}$ and $\lambda \in P_{+}^{(k)}$, let $L_{\lambda}^{(k)}=U(\widehat{\mathfrak{g}}) v_{\lambda}^{(k)}$ be the unique level $k$ integrable module such that

$$
\begin{aligned}
& \left(\mathfrak{g} \otimes t^{n}\right) v_{\lambda}^{(k)}=0 \quad(n>0), \\
& \mathfrak{n}_{+} v_{\lambda}^{(k)}=0, \\
& h v_{\lambda}^{(k)}=\langle\lambda, h\rangle v_{\lambda}^{(k)} \quad(h \in \mathfrak{h}), \quad d v_{\lambda}^{(k)}=0 .
\end{aligned}
$$

We also consider modules 'placed at infinity'. To be precise, define $\widehat{\mathfrak{g}}(\infty)=$ $\mathfrak{g} \otimes \mathbb{C}\left[t, t^{-1}\right] \oplus \mathbb{C} K \oplus \mathbb{C} d$ by the same equations as above, except that we change the cocycle to

$$
\begin{aligned}
\Omega_{\infty}(x \otimes f, y \otimes g) & =(x \mid y) \operatorname{Res}_{t=\infty} d f \cdot g \\
& =-\Omega_{0}(x \otimes f, y \otimes g) .
\end{aligned}
$$

Let $L_{\lambda}^{(k)}(\infty)=U(\widehat{\mathfrak{g}}(\infty)) v_{\lambda}^{(k)}(\infty)$ be the integrable $\widehat{\mathfrak{g}}(\infty)$-module characterized by (2.9)-(2.11), with $v_{\lambda}^{(k)}(\infty)$ in place of $v_{\lambda}^{(k)}$ and (2.9) being changed to

$$
\left(\mathfrak{g} \otimes t^{n}\right) v_{\lambda}^{(k)}(\infty)=0 \quad(n<0) .
$$

The degree zero part of $L_{\lambda}^{(k)}$ and $L_{\lambda}^{(k)}(\infty)$ are both isomorphic to $\pi_{\lambda}$ as $\mathfrak{g}$-module.

Consider the isomorphism of Lie algebra $\iota: \widehat{\mathfrak{g}} \rightarrow \widehat{\mathfrak{g}}(\infty)$ given by $x \otimes t^{n} \mapsto$ $x \otimes t^{n}, K \mapsto-K, d \mapsto d$. By this isomorphism, $L_{\lambda}^{(k)}(\infty)$ can be considered 
as the level $-k$ lowest weight module with the lowest weight $-\lambda$. We have a non-degenerate pairing

$$
L_{\lambda}^{(k)}(\infty) \times L_{\lambda^{*}}^{(k)} \longrightarrow \mathbb{C}
$$

such that $\langle\iota(x) u, v\rangle=-\langle u, x v\rangle\left(x \in \widehat{\mathfrak{g}}, u \in L_{\lambda}^{(k)}(\infty), v \in L_{\lambda^{*}}^{(k)}\right)$, which extends the canonical coupling $\pi_{\lambda} \times \pi_{\lambda^{*}} \rightarrow \mathbb{C}$.

Proposition 2.5. Let $X \subset U(\mathfrak{g}[t])$ be a congruence right ideal. Then $L_{\lambda}^{(k)}(\infty) / X$ is finite-dimensional. We have an isomorphism

$$
\left(L_{\lambda}^{(k)}(\infty) / X\right)^{*} \simeq\left(L_{\lambda^{*}}^{(k)}\right)^{S(X)} .
$$

Proof. First we prove the finite dimensionality of $L_{\lambda}^{(k)}(\infty) / X$. Choose a set of nilpotent generators $x_{1}, \cdots, x_{m}$ of $\mathfrak{g}$, and let $\widetilde{B}_{M, i}$ be the right ideal generated by $x_{j} \otimes t^{n}(1 \leq j \leq m, n \geq M)$ and $x_{1} \otimes t^{M-1}, \cdots, x_{i} \otimes t^{M-1}$. Note that $\widetilde{B}_{M, 0}=\widetilde{B}_{M+1, m}$. We have $\widetilde{B}_{M, i}=\widetilde{B}_{M, i-1}+\left(x_{i} \otimes t^{M-1}\right) U(\mathfrak{g}[t])$. Since the congruence ideal $X$ contains $\widetilde{B}_{M, 0}$ for a large $M$, in order to show the finite-dimensionality of $L_{\lambda}^{(k)}(\infty) / X$, it suffices to prove it for $L_{\lambda}^{(k)}(\infty) / \widetilde{B}_{M, i}$ for all $M, i$. Since $L_{\lambda}^{(k)}(\infty) / \widetilde{B}_{1,0} \simeq \pi_{\lambda}$, the statement is valid for $M=1, i=$ 0 . The general case can be shown by induction on $M, i$, by applying the following lemma to $V=L_{\lambda}^{(k)}(\infty) / \widetilde{B}_{M, i-1}, x=x_{i} \otimes t^{M-1}$.

Eq.(2.12) states that $L_{\lambda^{*}}^{(k)}$ is the restricted dual of $L_{\lambda}^{(k)}(\infty)$. Since $X$ is graded and $L_{\lambda}^{(k)}(\infty) / X$ is finite-dimensional, (2.13) follows from (2.7).

Lemma 2.6. Let $V$ be a vector space and $x \in \operatorname{End}(V)$. Assume that $x$ is locally nilpotent, $\bigcap_{n>0} \operatorname{Im} x^{n}=\{0\}$, and that $V / \operatorname{Im} x$ is finite dimensional. Then $V$ is also finite dimensional.

Proof. Choose vectors $v_{1}, \cdots, v_{n} \in V$ which span $V / \operatorname{Im} x$, and let $M$ be such that $x^{M} v_{i}=0$ for all $i$. Then the set $\left\{x^{j} v_{i}\right\}$ with $0 \leq j \leq M-1,1 \leq i \leq n$ spans $V$.

2.3. The multiplicative formula for dimensions. The aim of this section is to prove the multiplicative formula for the dimension of the space of coinvariants with respect to the fusion right ideals. Before discussing it, we recall the original version of the fusion rule for the space of conformal coinvariants.

From now on, we fix a level $k \in \mathbb{Z}_{\geq 0}$. First recall the definition of the Verlinde algebra. Let $\operatorname{Rep} \mathfrak{g}=\oplus_{\lambda \in P_{+}} \mathbb{Z} \cdot[\lambda]$ be the Grothendieck ring of $\mathfrak{g}$, where $[\lambda]$ stands for the class of irreducible representation $\pi_{\lambda}$. Let $\mathcal{R}^{(k)}$ denote the ideal spanned by elements of the form

$$
[\lambda]-(-1)^{l(w)}[w(\lambda, k)] \quad\left(w \in W_{\text {aff }}, \lambda, w(\lambda, k) \in P_{+}\right),
$$

where $l(w)$ signifies the length of $w$. The Verlinde algebra is the quotient ring $\mathcal{V}^{(k)}=\operatorname{Rep} \mathfrak{g} / \mathcal{R}^{(k)}$. We use the same letter $[\lambda]$ to represent its class 
in $\mathcal{V}^{(k)}$. The set $\{[\lambda]\}_{\lambda \in P_{+}^{(k)}}$ constitutes a $\mathbb{Z}$-basis of $\mathcal{V}^{(k)}$. For an element $a \in \mathcal{V}^{(k)}$, we write the coefficient of $[\lambda]$ in this basis as $(a:[\lambda])_{k}$, i.e.,

$$
a=\sum_{\lambda \in P_{+}^{(k)}}(a:[\lambda])_{k}[\lambda] .
$$

We now fix a set of distinct points $z=\left\{z_{1}, \cdots, z_{N}\right\}$ in $\mathbb{C}$.

Theorem 2.7. [TUY] Let $L_{i}(i=0, \ldots, N)$ be the irreducible highest weight representation of highest weight $\lambda_{i}$. Define the space of conformal coinvariants by (1.9). Then, we have the dimension formula.

$$
\operatorname{dim}\left\langle L_{0}, \ldots, L_{N}\right\rangle_{\infty, z_{1}, \ldots, z_{N}}^{\mathbb{C} P^{1}}=\left(\left[\lambda_{1}^{*}\right] \cdots\left[\lambda_{N}^{*}\right]:\left[\lambda_{0}\right]\right)_{k} .
$$

Now, consider a set of congruence right ideals $X_{1}, \cdots, X_{N}$ of $U(\mathfrak{g}[t])$.

Lemma 2.8. The space of coinvariants

$$
L_{\lambda}^{(k)}(\infty) / X_{1} \circledast \cdots \circledast X_{N}(z)
$$

is finite dimensional.

Proof. A standard deformation argument (e.g. FKLMM1, Lemma 20) shows that $\operatorname{dim} L_{\lambda}^{(k)}(\infty) / B_{M, z} \leq \operatorname{dim} L_{\lambda}^{(k)}(\infty) / B_{N M}<\infty$, where we used Proposition 2.5. Since $X_{1} \circledast \cdots \circledast X_{N}(z) \supset B_{M, z}$ for some $M$, we obtain the assertion.

The main result of this section is the following.

Theorem 2.9. Notation being as above, we have

$$
\begin{gathered}
\sum_{\lambda \in P_{+}^{(k)}} \operatorname{dim}\left(L_{\lambda}^{(k)}(\infty) / X_{1} \circledast \ldots \circledast X_{N}(Z)\right) \cdot[\lambda] \\
=\prod_{i=1}^{N}\left(\sum_{\lambda \in P_{+}^{(k)}} \operatorname{dim}\left(L_{\lambda}^{(k)}(\infty) / X_{i}\right) \cdot[\lambda]\right) .
\end{gathered}
$$

For the proof, we make use of a construction due to [FFu which we recall below. See also appendix to [FKLMM1, where a detailed account is given in the case $\mathfrak{g}=\mathfrak{s l}_{2}$.

Set

$$
\mathcal{C}_{\lambda, M}^{(k)}(z)=L_{\lambda}^{(k)}(\infty) / B_{M, z}
$$

where $B_{M, z}$ is defined in (2.3). Since $B_{M, z}$ is a two-sided ideal, $\mathrm{e}_{\lambda, M}^{(k)}(z)$ is a $U(\mathfrak{g}[t])$ module. Taking the dual module, we obtain an inductive system $\cdots \subset\left(\mathcal{C}_{\lambda, M}^{(k)}(\mathcal{Z})\right)^{*} \subset\left(\mathcal{C}_{\lambda, M+1}^{(k)}(\mathcal{Z})\right)^{*} \subset \cdots$. The direct limit

$$
\mathcal{L}_{\lambda}^{(k)}(\mathcal{z})=\underset{M}{\lim }\left(\mathcal{e}_{\lambda, M}^{(k)}(\mathcal{z})^{*}\right)
$$


admits an action of the algebra

$$
U_{Z}(\mathfrak{g}[t])=\lim _{M} U(\mathfrak{g}[t]) / B_{M, Z} \cdot
$$

Note that, in the special case where $z=\{0\}$,

$$
U_{\{0\}}(\mathfrak{g}[t])=\lim _{M} U(\mathfrak{g}[t]) /\left(t^{M} \mathfrak{g}[t] U(\mathfrak{g}[t])\right)
$$

is the completion by formal power series in $t$. We denote the completion of $U(\mathfrak{g}[t])^{\otimes N}$ as

$$
\begin{aligned}
& U_{\{0\}}(\mathfrak{g}[t])^{\widehat{\otimes} N} \\
& =\lim _{M} U(\mathfrak{g}[t])^{\otimes N} / I^{(N)}\left(B_{M}, \ldots, B_{M}\right) \text {. }
\end{aligned}
$$

We use the following lemmas.

Lemma 2.10. Suppose $\mathfrak{b}$ is an ideal of a Lie algebra $\mathfrak{a}$. Then, we have

$$
U(\mathfrak{a} / \mathfrak{b}) \simeq U(\mathfrak{a}) / \mathfrak{b} U(\mathfrak{a}) .
$$

Lemma 2.11. Suppose $\mathfrak{a}=\mathfrak{a}_{1} \oplus \cdots \oplus \mathfrak{a}_{N}$ is a direct sum decomposition of a Lie algebra. Then, we have an isomorphism of algebras

$$
U(\mathfrak{a}) \simeq U\left(\mathfrak{a}_{1}\right) \otimes \cdots \otimes U\left(\mathfrak{a}_{N}\right) .
$$

Now, we state a basic proposition about localization of the universal enveloping algebra.

Proposition 2.12. (i) We have a canonical isomorphism

$$
U_{z}(\mathfrak{g}[t]) \simeq U_{\{0\}}(\mathfrak{g}[t])^{\widehat{\otimes} N} .
$$

(ii) The canonical inclusion $U=U(\mathfrak{g}[t]) \rightarrow U_{\mathcal{Z}}(\mathfrak{g}[t]) \simeq U_{\{0\}}(\mathfrak{g}[t])^{\widehat{\otimes} N}$ is the composition of $\Delta_{z}$ followed by the inclusion $U(\mathfrak{g}[t])^{\otimes N} \rightarrow U_{\{0\}}(\mathfrak{g}[t])^{\widehat{\otimes} N}$.

(iii) The action of $U^{\otimes N}$ on $\mathcal{L}_{\lambda}^{(k)}(\mathcal{Z})$ can be extended to an action of $U(\widehat{\mathfrak{g}})^{\otimes N}$.

Proof. Since the points in $z$ are distinct, the restriction of $\Delta_{z}$ to $\mathfrak{g}[t]$ induces an isomorphism

$$
\iota_{\mathcal{Z}}: \mathfrak{g}[t] / \prod_{i=1}^{N}\left(t-z_{i}\right)^{M} \mathfrak{g}[t] \simeq \oplus^{N}\left(\mathfrak{g}[t] / t^{M} \mathfrak{g}[t]\right) .
$$

By Lemma 2.11 we have

$$
\Delta_{z}: U\left(\mathfrak{g}[t] / \prod_{i=1}^{N}\left(t-z_{i}\right)^{M} \mathfrak{g}[t]\right) \stackrel{\simeq}{\longrightarrow} U\left(\mathfrak{g}[t] / t^{M} \mathfrak{g}[t]\right)^{\otimes N}
$$

By Lemma 2.10, we have

$$
U / B_{M, z} \simeq\left(U / t^{M} \mathfrak{g}[t] U\right)^{\otimes N}
$$


For (i) and (ii), it is enough to show that the kernel of the canonical surjection $U^{\otimes N} \rightarrow\left(U / t^{M} \mathfrak{g}[t] U\right)^{\otimes N}$ coincides with $I^{(N)}\left(B_{M}, \ldots, B_{M}\right)$. This follows from Lemma 2.3 .

The action of $U(\widehat{\mathfrak{g}})^{\otimes N}$ is defined as follows. Consider an element $x(t)=$ $\left(x_{1}(t), \cdots, x_{N}(t)\right)$ of $\oplus^{N} \widehat{\mathfrak{g}}$. Choose an $m$ such that $t^{m} x_{i}(t) \in \mathfrak{g}[t]$ for all $i$. Then $\prod_{j=1}^{N}\left(t-z_{j}\right)^{m} \iota_{\mathcal{z}}^{-1}(x(t)) \in \mathfrak{g}[t]$, so that $\iota_{\mathcal{Z}}^{-1}(x(t))$ maps $\mathcal{C}_{\lambda, M}^{(k)}(\mathcal{Z})$ to $\mathcal{C}_{\lambda, M-m}^{(k)}(\mathcal{Z})$ for $M \geq m$. Therefore $x(t)$ acts on $\mathcal{L}_{\lambda}^{(k)}(\mathcal{Z})$.

We will use the following fact.

Theorem 2.13. [FFu We have an isomorphism of $U(\widehat{\mathfrak{g}})^{\otimes N}$-modules.

$$
\mathcal{L}_{\lambda}^{(k)}(z) \simeq \bigoplus_{\lambda_{1}, \ldots, \lambda_{N} \in P_{+}^{(k)}}\left(\left[\lambda_{1}\right] \cdots \cdot\left[\lambda_{N}\right]:[\lambda]\right)_{k} \cdot L_{\lambda_{1}^{*}}^{(k)} \otimes \cdots \otimes L_{\lambda_{N}^{*}}^{(k)} .
$$

For $I \subset U(\mathfrak{g}[t])$, denote by $I^{z}$ its closure in $U_{z}(\mathfrak{g}[t])$ in the topology of the inverse limit.

\section{Lemma 2.14 .}

$$
\left(L_{\lambda}^{(k)}(\infty) / X_{1} \circledast \ldots \circledast X_{N}(z)\right)^{*} \simeq\left(\mathcal{L}_{\lambda}^{(k)}(z)\right)^{S\left(X_{1} \circledast \ldots \circledast X_{N}(z)\right)^{z}} .
$$

Proof. Choose $M$ such that $X_{i} \supset B_{M}$ for all $i$. Then

$$
L_{\lambda}^{(k)}(\infty) / X_{1} \circledast \ldots \circledast X_{N}(z) \simeq \mathcal{C}_{\lambda, M}^{(k)}(z) / X_{1} \circledast \ldots \circledast X_{N}(z) .
$$

We have

$$
\left(\mathrm{e}_{\lambda, M}^{(k)}(\mathrm{z}) / X_{1} \circledast \ldots \circledast X_{N}(z)\right)^{*} \simeq\left(\mathrm{e}_{\lambda, M}^{(k)}(\mathrm{z})^{*}\right)^{S\left(X_{1} \circledast \ldots \circledast X_{N}(z)\right)} .
$$

Taking the direct limit in $M$ we obtain the statement of the lemma.

Lemma 2.15. Suppose that $z_{1}, \ldots, z_{N} \in \mathbb{C}$ are distinct. For any $L>0$ and $i=1, \cdots, N$, there exists an $f_{i}(t) \in \mathbb{C}[t]$ satisfying

$$
f_{i}(t) \equiv\left\{\begin{array}{l}
1 \bmod \left(t-z_{i}\right)^{L} ; \\
0 \bmod \left(t-z_{j}\right)^{L}
\end{array} \quad \text { for } j \neq i .\right.
$$

Lemma 2.16. Under the identification of Proposition 2.19 we have

$$
\left(X_{1} \circledast \ldots \circledast X_{N}(z)\right)^{\mathcal{Z}} \simeq \sum_{i=1}^{N} U_{\{0\}}(\mathfrak{g}[t])^{\widehat{\otimes}(i-1)} \widehat{\otimes} X_{i}^{\{0\}} \widehat{\otimes} U_{\{0\}}(\mathfrak{g}[t])^{\widehat{\otimes}(N-i)} .
$$

Here, the right hand side is the completion in (2.10).

Proof. For a sufficiently large $L$, we have

$$
I^{(N)}\left(B_{L}, \ldots, B_{L}\right) \subset I^{(N)}\left(X_{1}, \ldots, X_{N}\right) .
$$

Recall that $X_{1} \circledast \ldots \circledast X_{N}(Z)=\Delta_{Z}^{-1}\left(I^{(N)}\left(X_{1}, \ldots, X_{N}\right)\right)$. 
We want to show that for a sufficiently large $L$ and for any element $a \in$ $I^{(N)}\left(X_{1}, \ldots, X_{N}\right)$, there exists an element $x \in X_{1} \circledast \ldots \circledast X_{N}(Z)$ satisfying

$$
\Delta_{z}(x) \equiv a \bmod I^{(N)}\left(B_{L}, \ldots, B_{L}\right) .
$$

From (2.17) it is enough to find $x \in U(\mathfrak{g}[t])$ satisfying (2.18) because it implies $x \in X_{1} \circledast \ldots \circledast X_{N}(Z)$. The existence of such an $x$ follows from

$$
\Delta_{z}\left(f_{i}(t) g\right) \equiv 1 \otimes \cdots \otimes \stackrel{i-\text { th }}{g} \otimes \cdots \otimes 1 \bmod I^{(N)}\left(B_{L}, \ldots, B_{L}\right),
$$

where $g \in \mathfrak{g}[t]$ and $f_{i}(t)$ is given in Lemma 2.15.

Proof of Theorem 2.9. Set $X=X_{1} \circledast \ldots \circledast X_{N}(Z)$. Collecting together the statements above, we find

$$
\begin{aligned}
& \left(L_{\lambda}^{(k)}(\infty) / X\right)^{*} \simeq\left(\mathcal{L}_{\lambda}^{(k)}(Z)\right)^{S(X)^{z}} \quad \text { (by Lemma 2.14) } \\
& \simeq \bigoplus_{\lambda_{1}, \cdots, \lambda_{N} \in P_{+}^{(k)}}\left(\left[\lambda_{1}\right] \ldots\left[\lambda_{N}\right]:[\lambda]\right)_{k} \cdot\left(L_{\lambda_{1}^{*}}^{(k)} \otimes \cdots \otimes L_{\lambda_{N}^{*}}^{(k)}\right)^{S(X)^{z}}
\end{aligned}
$$

(by Theorem 2.13)

$$
\simeq \bigoplus_{\lambda_{1}, \cdots, \lambda_{N} \in P_{+}^{(k)}}\left(\left[\lambda_{1}\right] \ldots\left[\lambda_{N}\right]:[\lambda]\right)_{k} \cdot\left(L_{\lambda_{1}^{*}}^{(k)}\right)^{S\left(X_{1}\right)} \otimes \cdots \otimes\left(L_{\lambda_{N}^{*}}^{(k)}\right) S\left(X_{N}\right)
$$

(by Lemma 2.16)

$$
\begin{aligned}
& \simeq \bigoplus_{\lambda_{1}, \ldots, \lambda_{N} \in P_{+}^{(k)}}\left(\left[\lambda_{1}\right] \ldots\left[\lambda_{N}\right]:[\lambda]\right)_{k} \\
& \times\left(L_{\lambda_{1}}^{(k)}(\infty) / X_{1} \otimes \cdots \otimes L_{\lambda_{N}}^{(k)}(\infty) / X_{N}\right)^{*}
\end{aligned}
$$

(by Proposition 2.5).

Therefore

$$
\operatorname{dim} L_{\lambda}^{(k)}(\infty) / X=\sum_{\lambda_{1}, \ldots, \lambda_{N} \in P_{+}^{(k)}}\left(\left[\lambda_{1}\right] \cdots\left[\lambda_{N}\right]:[\lambda]\right)_{k} \cdot \prod_{i=1}^{N} \operatorname{dim} L_{\lambda_{i}}^{(k)}(\infty) / X_{i},
$$

which proves the theorem.

2.4. Space of conformal coinvariants. We give a sketch of proof for the surjectivity statements in Introduction, which motivate the definition of spaces of coinvariants in two different settings. In Section 3 these two spaces will be identified. We also conclude Section 2 by stating the isomorphism between the space of conformal coinvariants and the space of coinvariants by the fusion right ideal.

The proof of the surjectivity of (1.3) and (1.6) is based on the equality (1.4) where we take $x \in \mathfrak{g}^{C \backslash\left\{p_{0}, \ldots, p_{N}\right\}}$. There exists a meromorphic function $f$ on the curve $C$ which has poles only at $p_{0}$ and $p_{i}(i \neq 0)$. Moreover, the 
singular part of the pole at $p_{i}$ can be chosen arbitrarily. By using (1.4) for $x=X \otimes f(X \in \mathfrak{g})$, one can prove inductively the surjectivity of the map

$$
L_{0} \otimes \pi\left(L_{1}\right) \otimes \cdots \otimes \pi\left(L_{N}\right) \rightarrow\left\langle L_{0}, \ldots, L_{N}\right\rangle_{p_{0}, \ldots, p_{N}}^{C} .
$$

Now, for (1.3), taking $f$ which has a pole only at $p_{0}$ and such that $f\left(p_{j}\right)=$ $\delta_{i, j}$, we can reduce the vectors in $\pi\left(L_{i}\right)$ to the highest weight vector $v_{i}$. For (1.6), we assume that the curve $C$ is rational. Then, taking $f$ which has a pole only at $p_{0}$ (we can choose an arbitrary singular part of the pole), we can reduce the vectors in $L_{0}$ to the highest weight vector $v_{0}$.

Note that in the above proof we used only the property of the highest weight vector $v_{i}$ that it is annihilated by $\mathfrak{g} \otimes t_{i} \mathbb{C}\left[t_{i}\right]$ and that it is a cyclic vector in $L_{i}$. Therefore, we can replace $L_{i}$ by a direct sum of highest weight representations with a cyclic vector $v_{i}$ in the degree (with respect to $d$ ) zero component. The surjectivity (1.3) is still valid.

For $\lambda \in P_{+}$, we define the left ideal $\mathfrak{X}_{\lambda} \subset U(\mathfrak{g})$ by

$$
\mathfrak{X}_{\lambda}=U(\mathfrak{g}) \mathfrak{n}_{+}+\sum_{h \in \mathfrak{h}} U(\mathfrak{g})(h-\langle\lambda, h\rangle) .
$$

We also define $\mathfrak{X}_{\lambda}^{\prime} \subset U(\mathfrak{g})$ by

$$
\mathfrak{X}_{\lambda}^{\prime}=U(\mathfrak{g}) \mathfrak{n}_{+}+\sum_{h \in \mathfrak{h}} U(\mathfrak{g})(h-\langle\lambda, h\rangle)+\sum_{j} U(\mathfrak{g}) f_{j}^{\left\langle\lambda, \alpha_{j}^{\vee}\right\rangle+1}
$$

where $\alpha_{j}$ and $\alpha_{j}^{\vee}$ denote the simple roots and coroots respectively, and the $f_{j}$ are root vectors corresponding to $-\alpha_{j}$. Namely $\mathfrak{X}_{\lambda}^{\prime}$ is the annihilating ideal of the highest weight vector $v_{\lambda} \in \pi_{\lambda}$.

Lemma 2.17. We have

$$
\operatorname{dim} \pi_{\mu} / S\left(\mathfrak{X}_{\lambda}\right)=\operatorname{dim} \pi_{\mu} / S\left(\mathfrak{X}_{\lambda}^{\prime}\right)=\delta_{\mu, \lambda^{*}} .
$$

Proof. Let $\mathfrak{X}$ be $\mathfrak{X}_{\lambda}$ or $\mathfrak{X}_{\lambda}^{\prime}$.

Then the right ideal $S(\mathfrak{X})$ contains $\mathfrak{n}_{+}$and $h+\langle\lambda, h\rangle(h \in \mathfrak{h})$. Since $-\lambda=w_{0}\left(\lambda^{*}\right)$, this implies $\pi_{\mu} / S(\mathfrak{X})=0$ unless $\mu=\lambda^{*}$.

For $\mu=\lambda^{*}$, denote by $v_{\lambda}^{*}$ the lowest weight vector of $\pi_{\lambda^{*}}$ such that $\left\langle v_{\lambda}^{*}, v_{\lambda}\right\rangle=1$ with the highest weight vector $v_{\lambda} \in \pi_{\lambda}$. We have $\pi_{\lambda^{*}} / S(\mathfrak{X})=$ $\mathbb{C} v_{\lambda}^{*}$ if one can show that $v_{\lambda}^{*}$ is not contained in the image of $S(\mathfrak{X})$. Suppose that $v_{\lambda}^{*}=S(g) w$ for some $g \in \mathfrak{X}$ and $w \in \pi_{\lambda^{*}}$. Then, we have

$$
1=\left\langle v_{\lambda}^{*}, v_{\lambda}\right\rangle=\left\langle S(g) w, v_{\lambda}\right\rangle=\left\langle w, g v_{\lambda}\right\rangle=0
$$

as elements of $\mathfrak{X}$ annihilate the highest weight vector. This is a contradiction.

Corollary 2.18. Set

$$
X_{\lambda_{i}}=S\left(\mathfrak{X}_{\lambda_{i}}\right)+B_{1}, \quad X_{\lambda_{i}}^{\prime}=S\left(\mathfrak{X}_{\lambda_{i}}^{\prime}\right)+B_{1}
$$

for $\lambda_{i} \in P_{+}^{(k)}$. Then, we have

$$
\operatorname{dim} L_{\lambda}^{(k)}(\infty) / X_{\lambda_{1}} \circledast \ldots \circledast X_{\lambda_{N}}(Z)=\left(\left[\lambda_{1}^{*}\right] \cdots\left[\lambda_{N}^{*}\right]:[\lambda]\right)_{k},
$$




$$
\operatorname{dim} L_{\lambda}^{(k)}(\infty) / X_{\lambda_{1}}^{\prime} \circledast \ldots \circledast X_{\lambda_{N}}^{\prime}(Z)=\left(\left[\lambda_{1}^{*}\right] \cdots\left[\lambda_{N}^{*}\right]:[\lambda]\right)_{k} .
$$

Proof. As above, let $\mathfrak{X}$ be $\mathfrak{X}_{\lambda}$ or $\mathfrak{X}_{\lambda}^{\prime}$ and let $X$ be $X_{\lambda}$ or $X_{\lambda}^{\prime}$ respectively. Then

$$
L_{\lambda}^{(k)}(\infty) / X=L_{\lambda}^{(k)}(\infty) /\left(B_{1}+S(\mathfrak{X})\right) \simeq \pi_{\lambda} / S(\mathfrak{X}) .
$$

The statement follows from Lemma 2.17 and Theorem 2.9.

From Corollary 2.18 we have

Proposition 2.19. Let $z_{1}, \cdots, z_{N}$ be distinct points in $\mathbb{C}$. Set

$$
X_{\lambda_{i}}=S\left(\mathfrak{X}_{\lambda_{i}}\right)+B_{1}, \quad X_{\lambda_{i}}^{\prime}=S\left(\mathfrak{X}_{\lambda_{i}}^{\prime}\right)+B_{1}
$$

(i) We have the canonical isomorphism given by (1.3)

$$
L_{\lambda}^{(k)}(\infty) / X_{\lambda_{1}}^{\prime} \circledast \cdots \circledast X_{\lambda_{N}}^{\prime}(Z) \simeq\left\langle L_{\lambda}^{(k)}, L_{\lambda_{1}}^{(k)}, \ldots, L_{\lambda_{N}}^{(k)}\right\rangle_{\infty, z_{1}, \ldots, z_{N}}^{\mathbb{C} P^{1}}
$$

(ii) We also have the canonical isomorphism

$$
L_{\lambda}^{(k)}(\infty) / X_{\lambda_{1}} \circledast \cdots \circledast X_{\lambda_{N}}(Z) \simeq\left\langle L_{\lambda}^{(k)}, L_{\lambda_{1}}^{(k)}, \ldots, L_{\lambda_{N}}^{(k)}\right\rangle_{\infty, z_{1}, \ldots, z_{N}}^{\mathbb{C} P^{1}}
$$

Proof. First let us prove (i). The map (1.3) gives a surjection from the left hand side to the right hand side of $(2.21)$. The injectivity follows since their dimensions are equal by Theorem 2.9 and Corollary 2.18.

To prove (ii) note that as $X_{\lambda} \subset X_{\lambda}^{\prime}$ we have the natural surjection

$$
L_{\lambda}^{(k)}(\infty) / X_{\lambda_{1}} \circledast \cdots \circledast X_{\lambda_{N}}(z) \rightarrow L_{\lambda}^{(k)}(\infty) / X_{\lambda_{1}}^{\prime} \circledast \cdots \circledast X_{\lambda_{N}}^{\prime}(z) .
$$

Theorem 2.9 and Corollary 2.18 imply that the dimensions of these spaces are equal, so (2.23) and therefore (2.22) is an isomorphism.

\section{SPACES OF COINVARIANTS IN TERMS OF FILTERED TENSOR PRODUCT}

In this section we establish a connection between spaces of coinvariants (2.15) and the filtered tensor product $\mathcal{F}_{z}$ of finite dimensional $\mathfrak{g}$-modules introduced in [FL]. Motivated by Corollary 2.18, we restrict our considerations to right ideals $X_{1}, \cdots, X_{N}$ containing $B_{1}$, so that

$$
X_{i}=S\left(\mathfrak{X}_{i}\right)+B_{1}
$$

where $\mathfrak{X}_{i}$ is a left ideal of $U(\mathfrak{g})$. The main statement is Theorem 3.6.

3.1. Annihilating ideals. For a $\mathfrak{g}$-module $V$, we denote by $\operatorname{Ann} V \subset U(\mathfrak{g})$ the kernel of the structure map $U(\mathfrak{g}) \rightarrow \operatorname{End}(V)$. Ann $V$ is a two-sided ideal and $S(\operatorname{Ann} V)=\operatorname{Ann} V^{*}$. If $V$ is irreducible, then $U(\mathfrak{g}) / \operatorname{Ann} V \simeq \operatorname{End}(V) \simeq$ $V^{*} \otimes V$. The left $\mathfrak{g}$-action (resp., the right $\mathfrak{g}$-action) on $U(\mathfrak{g}) /$ Ann $V$ translates to the left $\mathfrak{g}$-action on $V$ (resp., the right $\mathfrak{g}$-action on $V^{*}$ ).

The representation $L_{\lambda}^{(k)}(\infty)$ is a quotient of $U(\mathfrak{g}[t])$ by the left ideal which describes the conditions on the highest weight vector $v_{\lambda}^{(k)}$. Hence the space

of coinvariants may be viewed as a bi-quotient of the algebra $U(\mathfrak{g}[t])$. More 
precisely, let $e_{\theta} \in \mathfrak{n}_{+}$be the root vector for the maximal root $\theta$. Introduce the following left ideals of $U(\mathfrak{g}[t])$, which annihilate $v_{\lambda}^{(k)}$ :

$$
\begin{aligned}
& I_{\lambda}=U(\mathfrak{g}[t]) \otimes_{U(\mathfrak{g})} \mathfrak{X}_{\lambda}=U(\mathfrak{g}[t]) \mathfrak{n}_{+}+\sum_{h \in \mathfrak{h}} U(\mathfrak{g}[t])(h-\langle\lambda, h\rangle), \\
& I_{\lambda}^{(k)}=I_{\lambda}+U(\mathfrak{g}[t])\left(e_{\theta} \otimes t\right)^{k-\left\langle\lambda, \theta^{\vee}\right\rangle+1} .
\end{aligned}
$$

These are not enough for the characterization of $v_{\lambda}^{(k)}$. However, we have

Lemma 3.1. We have

$$
U(\mathfrak{g}) /\left(\mathfrak{X}_{\lambda}+\operatorname{Ann} \pi_{\lambda}\right) \simeq \pi_{\lambda} .
$$

In other words, we have the equality of the left ideals (see (2.20))

$$
\mathfrak{X}_{\lambda}^{\prime}=\mathfrak{X}_{\lambda}+\operatorname{Ann} \pi_{\lambda} \text {. }
$$

Proof. We have

$$
U(\mathfrak{g}) / \operatorname{Ann} \pi_{\lambda} \simeq \pi_{\lambda}^{*} \otimes \pi_{\lambda} .
$$

Taking the quotient by the left ideal $\mathfrak{X}_{\lambda}$ we have

$$
\left(\pi_{\lambda}^{*} \otimes \pi_{\lambda}\right) /\left(\pi_{\lambda}^{*} \mathfrak{X}_{\lambda}\right) \otimes \pi_{\lambda} \simeq \mathbb{C} v_{\lambda}^{*} \otimes \pi_{\lambda} \simeq \pi_{\lambda} .
$$

We have the following characterization of $L_{\lambda}^{(k)}(\infty)$ as a quotient of $U(\mathfrak{g}[t])$.

Proposition 3.2. We have

$$
L_{\lambda}^{(k)}(\infty) \simeq U(\mathfrak{g}[t]) /\left(I_{\lambda}^{(k)}+U(\mathfrak{g}[t]) \operatorname{Ann} \pi_{\lambda}\right) .
$$

Proof. Using Lemma 3.1, we obtain

$$
\begin{aligned}
L_{\lambda}^{(k)}(\infty) & \simeq\left(U(\mathfrak{g}[t]) \otimes_{U(\mathfrak{g})} \pi_{\lambda}\right) / U(\mathfrak{g}[t])\left(e_{\theta} \otimes t\right)^{k-\left\langle\lambda, \theta^{\vee}\right\rangle+1} \\
& \simeq U(\mathfrak{g}[t]) \otimes_{U(\mathfrak{g})}\left(U(\mathfrak{g}) /\left(\mathfrak{X}_{\lambda}+\operatorname{Ann} \pi_{\lambda}\right)\right) / U(\mathfrak{g}[t])\left(e_{\theta} \otimes t\right)^{k-\left\langle\lambda, \theta^{\vee}\right\rangle+1} \\
& \simeq U(\mathfrak{g}[t]) /\left(I_{\lambda}^{(k)}+U(\mathfrak{g}[t]) \operatorname{Ann} \pi_{\lambda}\right) .
\end{aligned}
$$

Set

$$
\mathfrak{I}^{(k)}=\operatorname{Ann} \bigoplus_{\lambda \in P_{+}^{(k)}} \pi_{\lambda} .
$$

Clearly $\mathfrak{I}^{(k)}$ is a two-sided ideal and $S\left(\mathfrak{I}^{(k)}\right)=\mathfrak{I}^{(k)}$. We have an isomorphism of $U(\mathfrak{g})$-bimodules

$$
U(\mathfrak{g}) / \mathfrak{I}^{(k)} \simeq \bigoplus_{\lambda \in P_{+}^{(k)}} \pi_{\lambda}^{*} \otimes \pi_{\lambda} .
$$

For a left ideal $\mathfrak{X} \subset U(\mathfrak{g})$, introduce the left $\mathfrak{g}$-module

$$
\pi^{(k)}(\mathfrak{X})=\bigoplus_{\lambda \in P_{+}^{(k)}}\left(\pi_{\lambda}^{\mathfrak{X}}\right)^{*} \otimes \pi_{\lambda},
$$


and the left ideal

$$
\tilde{\mathfrak{X}}=\mathfrak{X}+\mathfrak{I}^{(k)} \subset U(\mathfrak{g}) .
$$

Proposition 3.3. We have

$$
\pi^{(k)}(\mathfrak{X}) \simeq U(\mathfrak{g}) / \tilde{\mathfrak{X}}
$$

In particular, $\pi^{(k)}(\mathfrak{X})$ is a cyclic $\mathfrak{g}$-module, the cyclic vector being the sum of canonical vectors of $\left(\pi_{\lambda}^{\mathfrak{X}}\right)^{*} \otimes \pi_{\lambda} \simeq \operatorname{Hom}_{\mathbb{C}}\left(\pi_{\lambda}^{\mathfrak{X}}, \pi_{\lambda}\right)$ corresponding to the inclusion $\pi_{\lambda}^{\mathfrak{X}} \subset \pi_{\lambda}$.

Proof. The isomorphism (3.5) is clear from (3.3):

$$
U(\mathfrak{g}) / \tilde{\mathfrak{X}} \simeq\left(U(\mathfrak{g}) / \mathfrak{I}^{(k)}\right) / \mathfrak{X} \simeq \bigoplus_{\lambda \in P_{+}^{(k)}}\left(\pi_{\lambda}^{*} / \pi_{\lambda}^{*} \mathfrak{X}\right) \otimes \pi_{\lambda} \simeq \pi^{(k)}(\mathfrak{X}) .
$$

Since $1 \in U(\mathfrak{g})$ is mapped in $(3.3)$ to the canonical vector in the right hand side, the statement about the cyclic vector follows.

The statement of Lemma 3.1 can be improved as follows.

Corollary 3.4. For $\lambda \in P_{+}^{(k)}$ we have

$$
U(\mathfrak{g}) /\left(\mathfrak{X}_{\lambda}+\mathfrak{I}^{(k)}\right) \simeq \pi^{(k)}\left(\mathfrak{X}_{\lambda}\right) \simeq \pi_{\lambda} .
$$

In other words, we have the equality of the left ideals

$$
\mathfrak{X}_{\lambda}^{\prime}=\mathfrak{X}_{\lambda}+\operatorname{Ann} \pi_{\lambda}=\mathfrak{X}_{\lambda}+\mathfrak{I}^{(k)}=\widetilde{\mathfrak{X}_{\lambda}}
$$

\subsection{Isomorphism with filtered tensor product.}

Proposition 3.5. Let $\tilde{\mathfrak{X}}_{i} \subset U(\mathfrak{g})$ be left ideals such that $U(\mathfrak{g}) / \tilde{\mathfrak{X}}_{i}$ is finite dimensional, $i=1, \cdots, N$. Define right ideals of $U(\mathfrak{g}[t])$ by $\tilde{X}_{i}=S\left(\tilde{\mathfrak{X}}_{i}\right)+B_{1}$. Then we have a canonical isomorphism

$$
U(\mathfrak{g}[t]) / \tilde{X}_{1} \circledast \cdots \circledast \tilde{X}_{N}(Z) \simeq \mathcal{F}_{\mathcal{Z}}\left(U(\mathfrak{g}) / \tilde{\mathfrak{X}}_{1}, \cdots, U(\mathfrak{g}) / \tilde{\mathfrak{X}}_{N}\right) .
$$

In this isomorphism the right action of an element $x \in U(\mathfrak{g}[t])$ in the left hand side is translated to the left action of $S(x)$ in the right hand side.

Proof. Set $U=U(\mathfrak{g}[t])$, and let $p_{i}: U \rightarrow U / \phi_{z_{i}}\left(\tilde{X}_{i}\right)$ be the projection. Consider the composition of maps

$$
\begin{aligned}
U \quad & \stackrel{\Delta(N-1)}{\longrightarrow} U \otimes \cdots \otimes U \\
& \stackrel{p_{1} \otimes \cdots \otimes p_{N}}{\longrightarrow} U / \phi_{z_{1}}\left(\tilde{X}_{1}\right) \otimes \cdots \otimes U / \phi_{z_{N}}\left(\tilde{X}_{N}\right) \\
& \stackrel{S \otimes \cdots \otimes}{\longrightarrow} U / \phi_{z_{1}}\left(\tilde{\mathfrak{X}}_{1}+B_{1}\right) \otimes \cdots \otimes U / \phi_{z_{N}}\left(\tilde{\mathfrak{X}}_{N}+B_{1}\right) .
\end{aligned}
$$

Since $U / \phi_{z_{i}}\left(\tilde{\mathfrak{X}}_{i}+B_{1}\right)$ is isomorphic to the evaluation module $U(\mathfrak{g}) / \tilde{\mathfrak{X}}_{i}$ at $t=z_{i}$, the last member is nothing but

$$
\mathcal{F}_{Z}\left(U(\mathfrak{g}) / \tilde{\mathfrak{X}}_{1}, \cdots, U(\mathfrak{g}) / \tilde{\mathfrak{X}}_{N}\right) .
$$

The map $U \rightarrow \mathcal{F}_{\mathcal{Z}}\left(U(\mathfrak{g}) / \tilde{\mathfrak{X}}_{1}, \cdots, U(\mathfrak{g}) / \tilde{\mathfrak{X}}_{N}\right)$ is surjective since the filtered tensor product is cyclic. 
Proposition is proved if we show that the kernel of $\left(p_{1} \otimes \cdots \otimes p_{N}\right) \circ \Delta^{(N-1)}$ coincides with $\tilde{X}_{1} \circledast \cdots \circledast \tilde{X}_{N}(Z)$. This is shown similarly as in the proof of Proposition 2.12 by using Lemma 2.3 .

We are now in a position to state the main result of this section.

Theorem 3.6. Let $\mathfrak{X}_{i} \subset U(\mathfrak{g})(i=1, \cdots, N)$ be a set of left ideals. Define right ideals of $U(\mathfrak{g}[t])$ by $X_{i}=S\left(\mathfrak{X}_{i}\right)+B_{1}$. Then we have a canonical isomorphism of filtered vector space

$$
L_{\lambda}^{(k)}(\infty) / X_{1} \circledast \ldots \circledast X_{N}(Z) \simeq \mathcal{F}_{z}\left(\pi^{(k)}\left(\mathfrak{X}_{1}\right), \ldots, \pi^{(k)}\left(\mathfrak{X}_{N}\right)\right) / S\left(I_{\lambda}^{(k)}\right)
$$

where $\pi^{(k)}(\mathfrak{X})$ is defined in (3.4) and the right ideal $S\left(I_{\lambda}^{(k)}\right)$ is given by (3.2):

$$
S\left(I_{\lambda}^{(k)}\right)=\mathfrak{n}_{+} U(\mathfrak{g}[t])+\sum_{h \in \mathfrak{h}}(h+\langle\lambda, h\rangle) U(\mathfrak{g}[t])+\left(e_{\theta} \otimes t\right)^{k-\left\langle\lambda, \theta^{\vee}\right\rangle+1} U(\mathfrak{g}[t]) .
$$

Proof. Set

$$
\tilde{\mathfrak{X}}_{i}=\mathfrak{X}_{i}+\mathfrak{I}^{(k)} .
$$

We have

$$
\tilde{X}_{i} \stackrel{\text { def }}{=} S\left(\tilde{\mathfrak{X}}_{i}\right)+B_{1}=X_{i}+\mathfrak{I}^{(k)} U(\mathfrak{g}[t]) .
$$

Since $\mathfrak{I}^{(k)}$ annihilates the $\mathfrak{g}$-module $L_{\lambda}^{(k)}(\infty) / B_{1}$, we have $L_{\lambda}^{(k)}(\infty) / X_{i} \simeq$ $L_{\lambda}^{(k)}(\infty) / \tilde{X}_{i}$. Comparing dimensions using Theorem 2.9, we find that the canonical projection

$$
L_{\lambda}^{(k)}(\infty) / X_{1} \circledast \ldots \circledast X_{N}(Z) \longrightarrow L_{\lambda}^{(k)}(\infty) / \tilde{X}_{1} \circledast \ldots \circledast \tilde{X}_{N}(Z)
$$

is an isomorphism.

We have

$$
\begin{aligned}
U(\mathfrak{g}[t]) / \tilde{X}_{1} \circledast \cdots \circledast \tilde{X}_{N}(\mathcal{Z}) \simeq & \mathcal{F}_{\mathcal{Z}}\left(U(\mathfrak{g}) / \tilde{\mathfrak{X}}_{1}, \cdots, U(\mathfrak{g}) / \tilde{\mathfrak{X}}_{N}\right) \\
& (\text { by Proposition 3.5) } \\
\simeq & \mathcal{F}_{Z}\left(\pi^{(k)}\left(\mathfrak{X}_{1}\right), \cdots, \pi^{(k)}\left(\mathfrak{X}_{N}\right)\right) .
\end{aligned}
$$

(by Proposition 3.3)

Therefore, from Proposition 3.2 follows that

$$
\begin{aligned}
& L_{\lambda}^{(k)}(\infty) / \tilde{X}_{1} \circledast \ldots \circledast \tilde{X}_{N}(\mathcal{Z}) \\
& \simeq \mathcal{F}_{Z}\left(\pi^{(k)}\left(\mathfrak{X}_{1}\right), \ldots, \pi^{(k)}\left(\mathfrak{X}_{N}\right)\right) /\left(S\left(I_{\lambda}^{(k)}\right)+\operatorname{Ann} \pi_{\lambda^{*}} U(\mathfrak{g}[t])\right) .
\end{aligned}
$$

The filtered tensor product $V=\mathcal{F}_{\mathcal{Z}}\left(\pi^{(k)}\left(\mathfrak{X}_{1}\right), \cdots, \pi^{(k)}\left(\mathfrak{X}_{N}\right)\right)$ is a finite dimensional $\mathfrak{g}$-module. Hence it has the form $V=\oplus_{\mu} V_{\mu}$ where $V_{\mu}$ is a direct sum of copies of $\pi_{\mu}$. Noting that $S\left(I_{\lambda}\right)=\mathfrak{n}_{+} U(\mathfrak{g}[t])+\sum_{h \in \mathfrak{h}}(h+\langle\lambda, h\rangle) U(\mathfrak{g}[t])$, we have $V / S\left(I_{\lambda}\right) \simeq V_{\lambda^{*}} / S\left(I_{\lambda}\right)$, and $\operatorname{Ann}\left(\pi_{\lambda^{*}}\right)$ acts as 0 on it. Therefore

$$
V /\left(S\left(I_{\lambda}^{(k)}\right)+\operatorname{Ann}\left(\pi_{\lambda^{*}}\right) U(\mathfrak{g}[t])\right) \simeq V / S\left(I_{\lambda}^{(k)}\right) .
$$

Combining (3.9), (3.10) and (3.11), we obtain the assertion. 
For filtered tensor product of irreducible modules, we obtain the following rule for the dimension.

Corollary 3.7. Suppose that $\lambda_{1}, \ldots, \lambda_{N} \in P_{+}^{(k)}$. Then, we have

$$
\operatorname{dim} \mathcal{F}_{Z}\left(\pi_{\lambda_{1}}, \cdots, \pi_{\lambda_{N}}\right) / S\left(I_{\lambda}^{(k)}\right)=\left(\left[\lambda_{1}^{*}\right] \cdots\left[\lambda_{N}^{*}\right]:[\lambda]\right)_{k} .
$$

Proof. Set $\mathfrak{X}_{i}=\mathfrak{X}_{\lambda_{i}}$. From Corollary 3.4 we have $\pi^{(k)}\left(\mathfrak{X}_{i}\right) \simeq \pi_{\lambda_{i}}$. Therefore, we have

$$
\mathcal{F}_{\mathcal{Z}}\left(\pi_{\lambda_{1}}, \cdots, \pi_{\lambda_{N}}\right) / S\left(I_{\lambda}^{(k)}\right) \simeq \mathcal{F}_{\mathcal{Z}}\left(\pi^{(k)}\left(\mathfrak{X}_{1}\right), \cdots, \pi^{(k)}\left(\mathfrak{X}_{N}\right)\right) / S\left(I_{\lambda}^{(k)}\right)
$$

Using Theorem 3.6, and applying Theorem 2.9 and Corollary 2.18, we have the assertion.

Example 1. For $\lambda_{i} \in P_{+}^{(k)}$, set $\mathfrak{X}_{i}=\mathfrak{X}_{\lambda_{i}}$ or $\mathfrak{X}_{i}=\mathfrak{X}_{\lambda_{i}}^{\prime}($ see $(2.19),(2.20)$ ). Then, from Corollary 3.4, we have

$$
L_{\lambda}^{(k)}(\infty) / X_{1} \circledast \cdots \circledast X_{N}(Z) \simeq \mathcal{F}_{Z}\left(\pi_{\lambda_{1}}, \cdots, \pi_{\lambda_{N}}\right) / S\left(I_{\lambda}^{(k)}\right) .
$$

By Proposition 2.19, we have an isomorphism to the space of conformal coinvariants on $\mathbb{C} P^{1}$ :

$$
L_{\lambda}^{(k)}(\infty) / X_{1} \circledast \cdots \circledast X_{N}(\mathcal{Z}) \simeq L_{\lambda} \otimes L_{\lambda_{1}} \otimes \cdots \otimes L_{\lambda_{N}} / \mathfrak{g}^{\mathbb{C} P^{1} \backslash\left\{\infty, z_{1}, \ldots, z_{N}\right\}} .
$$

Example 2. Let $\mathfrak{X}_{i}=0$ for all $i$. Then $\pi^{(k)}(0)=\sum_{\lambda \in P_{+}^{(k)}} \pi_{\lambda}^{*} \otimes \pi_{\lambda}$, and

$$
L_{\lambda}^{(k)}(\infty) / B_{1, \mathcal{Z}} \simeq \mathcal{F}_{\mathcal{Z}}\left(\pi^{(k)}(0), \cdots, \pi^{(k)}(0)\right) / S\left(I_{\lambda}^{(k)}\right) .
$$

The left hand side has dimension $\left(\left(\sum_{\mu \in P_{+}^{(k)}} \operatorname{dim} \pi_{\mu} \cdot[\mu]\right)^{N}:[\lambda]\right)_{k}$.

Example 3. Let $\mathfrak{X}_{i}=\mathfrak{X}=U(\mathfrak{g}) \mathfrak{n}_{+}$for all $i$. We choose the sum of highest weight vectors of $\pi_{\lambda}$ as cyclic vector of the module $\pi^{(k)}(\mathfrak{X}) \simeq \bigoplus_{\lambda \in P_{+}^{(k)}} \pi_{\lambda}$. Set $X=\mathfrak{n}_{+} U(\mathfrak{g})+B_{1}=\left(\mathfrak{n}_{+} \otimes \mathbb{C}[t] \oplus \mathfrak{h} \otimes t \mathbb{C}[t] \oplus \mathfrak{n}_{-} \otimes t \mathbb{C}[t]\right) U(\mathfrak{g}[t])$. From Proposition 2.1, the fusion right ideal is generated by the Lie subalgebra

$$
\mathfrak{a}(Z)=\mathfrak{n}_{+} \otimes \mathbb{C}[t] \oplus \mathfrak{h} \otimes \prod_{i=1}^{N}\left(t-z_{i}\right) \mathbb{C}[t] \oplus \mathfrak{n}_{-} \otimes \prod_{i=1}^{N}\left(t-z_{i}\right) \mathbb{C}[t] .
$$

Then, we have

$$
L_{\lambda}^{(k)}(\infty) / \mathfrak{a}(\mathcal{Z}) \simeq \mathcal{F}_{\mathcal{Z}}\left(\pi^{(k)}(\mathfrak{X}), \cdots, \pi^{(k)}(\mathfrak{X})\right) / S\left(I_{\lambda}^{(k)}\right) .
$$

The left hand side has dimension $\left(\left(\sum_{\mu \in P_{+}^{(k)}}[\mu]\right)^{N}:[\lambda]\right)_{k}$.

Example 4. Let $\mathfrak{g}=\mathfrak{s l}_{2}$ with standard generators $e, f, h$. Let $\pi_{l}(0 \leq$ $l \leq k)$ denote the $(l+1)$-dimensional irreducible representation, $[l]$ the corresponding element of $\mathcal{V}^{(k)}$, and $l$ the highest weight. For $0 \leq m \leq k$, set

$$
\begin{aligned}
& \mathfrak{Y}_{m}=U(\mathfrak{g}) e^{m+1}+U(\mathfrak{g}) f, \\
& \overline{\mathfrak{Y}}_{m}=U(\mathfrak{g}) f^{m+1}+U(\mathfrak{g}) e,
\end{aligned}
$$


and $Y_{m}=S\left(\mathfrak{Y}_{m}\right)+B_{1}, \bar{Y}_{m}=S\left(\overline{\mathfrak{Y}}_{m}\right)+B_{1}$. Then

$$
\pi^{(k)}\left(\mathfrak{Y}_{m}\right)=\oplus_{l=0}^{k}\left(\pi_{l}^{\mathfrak{Y} m}\right)^{*} \otimes \pi_{l} \simeq \oplus_{l=0}^{m} \pi_{l},
$$

with the cyclic vector being the sum of lowest weight vectors. Likewise $\pi^{(k)}\left(\overline{\mathfrak{Y}}_{m}\right) \simeq \oplus_{l=0}^{m} \pi_{l}$ has the sum of highest weight vectors as cyclic vector. Let $Y$ be the fusion right ideal of

$$
\overbrace{Y_{1}, \cdots, Y_{1}}^{m_{1}}, \cdots, \overbrace{Y_{k}, \cdots, Y_{k}}^{m_{k}}, \overbrace{\bar{Y}_{1}, \cdots, \bar{Y}_{1}}^{n_{1}}, \cdots, \overbrace{\bar{Y}_{k}, \cdots, \bar{Y}_{k}}^{n_{k}} .
$$

Then the space of coinvariants $L_{l}^{(k)}(\infty) / Y$ has dimension

$$
\left(([0]+[1])^{m_{1}+n_{1}} \cdots([0]+\cdots+[k])^{m_{k}+n_{k}}:[l]\right)_{k} .
$$

For $m=k$, one can replace $\mathfrak{Y}_{k}$ by $U(\mathfrak{g}) f$ without changing the space of coinvariants $(3.8)$ since $\pi^{(k)}\left(\mathfrak{Y}_{k}\right)=\pi^{(k)}(U(\mathfrak{g}) f)$. Similarly, one can replace $\overline{\mathfrak{Y}}_{k}$ by $U(\mathfrak{g}) e$. Therefore, instead of $Y_{k}$ and $\bar{Y}_{k}$, one can use

$$
\begin{aligned}
& Z=(e \otimes t \mathbb{C}[t] \oplus h \otimes t \mathbb{C}[t] \oplus f \otimes \mathbb{C}[t]) U(\mathfrak{g}), \\
& \bar{Z}=(e \otimes \mathbb{C}[t] \oplus h \otimes t \mathbb{C}[t] \oplus f \otimes t \mathbb{C}[t]) U(\mathfrak{g}),
\end{aligned}
$$

respectively.

Consider the special case where $m_{i}=n_{i}=0$ for $1 \leq i \leq k-1$ and $m_{k}=M, n_{k}=N$. In this case, the fusion right ideal

$$
\overbrace{Z \circledast \cdots \circledast Z}^{M} \circledast \overbrace{\bar{Z} \circledast \cdots \circledast \bar{Z}}^{N}\left(z_{1}, \ldots, z_{M}, z_{1}^{\prime}, \ldots, z_{N}^{\prime}\right)
$$

is generated by the Lie subalgebra

$$
\begin{aligned}
& \mathfrak{a}^{(M, N)}\left(Z, z^{\prime}\right) \\
& =e \otimes \prod_{i=1}^{M}\left(t-z_{i}\right) \mathbb{C}[t] \oplus h \otimes \prod_{i=1}^{M}\left(t-z_{i}\right) \prod_{i=1}^{N}\left(t-z_{i}^{\prime}\right) \mathbb{C}[t] \oplus f \otimes \prod_{i=1}^{N}\left(t-z_{i}^{\prime}\right) \mathbb{C}[t] .
\end{aligned}
$$

The space of coinvariants with respect to $\mathfrak{a}^{(M, N)}\left(\mathcal{Z}, \mathcal{Z}^{\prime}\right)$, in particular in the degeneration limit $z_{1}=\cdots=z_{M}=z_{1}^{\prime}=\cdots=z_{N}^{\prime}=0$, is the subject of the works [FKLMM2, FKLMM3].

3.3. Fusion Kostka polynomials. In this subsection, we first recall the definition of $q$-supernomials, Kostka polynomials and their generalizations from the literature, and then propose their counterparts in the theory of fusion product.

For two partitions $\lambda, \mu$ such that $|\lambda|=|\mu|$, the Kostka-Foulkes polynomials $K_{\lambda, \mu}(q)$ are the entries of the transition matrix from Schur functions $s_{\lambda}(x)$ to Hall-Littlewood functions $P_{\mu}(x ; q)$ (see [Mad] p. 239):

$$
s_{\lambda}(x)=\sum_{\mu} K_{\lambda, \mu}(q) P_{\mu}(x, q) .
$$

If $|\lambda| \neq|\mu|, K_{\lambda, \mu}(q)=0$. 
From the theory of Schur functions, it is known that the Kostka number $K_{\lambda, \mu}(1)$ is the cardinality of the set of semi-standard tableaux $\operatorname{Tab}(\lambda, \mu)$ of shape $\lambda$ and weight $\mu$. Therefore it was conjectured by [Fo that there should exist a charge statistic $c: \operatorname{Tab}(\lambda, \mu) \rightarrow \mathbb{Z}_{\geq 0}$ on semi-standard tableaux such that

$$
K_{\lambda, \mu}(q)=\sum_{t \in \operatorname{Tab}(\lambda, \mu)} q^{c(t)}
$$

This charge statistic was indeed found in [LS].

In the representation theory of $\mathfrak{g l}_{n}$, the Kostka number appears in two ways. In this connection, we have the restriction on the length of $\lambda: \ell(\lambda) \leq$ $n$. First, for $\mu$ satisfying $\ell(\mu) \leq n$, it is equal to the multiplicity of the weight $\mu$ in the $\mathfrak{g l}_{n}$ representation $V_{\lambda}$ with highest weight $\lambda$.

Secondly, for general $\mu$ such that $\ell(\mu)=N$, it is equal to the multiplicity of the $\mathfrak{g l}_{n}$-representation $\pi_{\lambda}$ in the tensor product of the $N$ symmetric tensor representations $V_{\left(\mu_{i}\right)}$ of $\mathfrak{g l}_{n}$ :

$$
\pi_{\left(\mu_{1}\right)} \otimes \cdots \otimes \pi_{\left(\mu_{N}\right)} \simeq \oplus_{\lambda: \ell(\lambda) \leq n} K_{\lambda, \mu}(1) \pi_{\lambda} .
$$

From the latter viewpoint, the Kostka polynomial can be regarded as the $q$-multiplicity of the representation $\pi_{\lambda}$ in the tensor product of symmetric tensor representations. Namely, it is the Hilbert polynomial in the variable $q$ of the graded space

$$
\operatorname{Hom}_{\mathfrak{g l}_{n}}\left(\pi_{\lambda}, \pi_{\left(\mu_{1}\right)} \otimes \cdots \otimes \pi_{\left(\mu_{N}\right)}\right),
$$

where the grading is given by the charge statistic.

This interpretation first appeared in [KR], where the completeness problem for Bethe ansatz solutions of the XXX model was considered. This is also the place where fermionic formulas for the Kostka polynomials first appeared. In that approach, the fermionic formulas were proven by constructing a (charge preserving) bijection between rigged configurations appearing in the completeness problem for the Bethe equations, and Young tableaux. The term "fermionic formulas" were invented in the works of Stony Brook group [KKMM], [KM], in which it was found that the Bethe ansatz solution gives, in the conformal limit, the characters of conformal field theory in fermionic form, where the $q$-grading is the linearized quasi-particle energy function. This gives a physical interpretation to the $q$-grading used in [KR].

Kostka polynomials were generalized [KS, SW1, SW2 to the case where the representations in the tensor product correspond to highest weight representations for $\mathfrak{s l}_{n}$ with highest weight $m \lambda_{i}$ (rectangular highest weight) where $\lambda_{i}(1 \leq i \leq n-1)$ are the fundamental weights.

There are two variants of Kostka polynomials: the $q$-supernomials and the level-restricted Kostka polynomials.

The $q$-supernomials are related to the Kostka polynomials by

$$
S_{\lambda, \mu}(q)=\sum_{\nu} K_{\nu, \lambda}(1) K_{\nu, \mu}(q)
$$


Namely, it is the $q$-multiplicity of the weight $\lambda$ in the tensor product (3.14). Fermionic formulas for the $q$-supernomial $S_{\lambda, \mu}(q)$ is known HKKOTY, and recursion equations for the generalized version in the sense of rectangular diagram are studied [SW2].

The level restriction has an origin in the corner transfer matrix method of Baxter. It was systematically studied as one-dimensional configuration sums in connection with the characters of integrable highest weight representations of affine Lie algebras. In this context, the level parameter appears as the level of representation. Kashiwara's theory of crystal base gave a solid basis to this approach. Quite generally, the crystal base of level $k$ integrable highest weight representations of affine Lie algebras are given in terms of paths, i.e., elements in semi-infinite product $B^{\otimes \infty / 2}$ of a finite dimensional affine crystal $B$. The $q$-statistics for paths are related to the eigenvalues of corner transfer matrix in the zero temperature limit.

The connection between Kostka polynomials and crystal base was discovered and generalized in [NY, SW1, SW2, HKKOTY]. In these works were considered finite and inhomogeneous paths, i.e., elements in a finite tensor product $B_{1} \otimes \cdots \otimes B_{N}$ of affine crystals $B_{i}$. There are actions of Kashiwara operators $\tilde{e}_{i}, \tilde{f}_{i}(i=0,1, \ldots, r)$ on affine crystals corresponding to the Chevalley generators $e_{i}, f_{i}$ of the affine Lie algebras. In this language, the generalized Kostka polynomial is the $q$-statistical sum over paths restricted by the highest weight conditions.

$$
\tilde{e}_{i}\left(b_{1} \otimes \cdots \otimes b_{N}\right)=0 \quad(i=1, \ldots, r) .
$$

The $q$-supernomial is nothing but the unrestricted sum, and the level $k$ restricted Kostka polynomial is the sum with the integrability conditions

$$
\tilde{f}_{0}^{k-\left\langle\lambda, \theta^{\vee}\right\rangle+1}\left(b_{1} \otimes \cdots \otimes b_{N}\right)=0 .
$$

For the generalized Kostka polynomials, fermionic formulas are known in the case of rectangular weights KSS. To our knowledge, no formulas are available at present for an arbitrary sequence of highest weights. Fermionic formulas for the level restricted (generalized) Kostka polynomials were obtained in SS] (see also HKKOTY]).

Now let us formulate the fusion product analogs of the Kostka and related polynomials. In what follows we consider only left ideals $\mathfrak{X} \subset U(\mathfrak{g})$ which are also $P$-graded,

$$
\mathfrak{X}=\oplus_{\nu \in P} \mathfrak{X}_{\nu},
$$

where $\mathfrak{X}_{\nu}=\{x \in \mathfrak{X} \mid[h, x]=\langle\nu, h\rangle x \forall h \in \mathfrak{h}\}$. The space of coinvariants are then $P$-graded, as well as filtered by the degree of $t$. In the isomorphism (3.7) of Proposition 3.5, the left action by $h \in \mathfrak{h}$ on the left hand side is translated to the right action by $S(h)=-h$ on the filtered tensor product (the right hand side). The latter commutes with the action of $\mathfrak{g}$, and is not to be confused with the left action of $h \in \mathfrak{g}$. To be concrete, let us take a basis $\left\{u_{\lambda, i}\right\}$ of $\pi_{\lambda}^{\mathfrak{X}}$ and the dual basis $\left\{u_{\lambda}^{i}\right\}$ of $\left(\pi_{\lambda}^{\mathfrak{X}}\right)^{*}$. The cyclic vector 
of $\pi^{(k)}(\mathfrak{X})$ is $u^{(k)}(\mathfrak{X})=\sum_{\lambda \in P_{+}^{(k)}} \sum_{i} u_{\lambda}^{i} \otimes u_{\lambda, i}$, and the right action of $S(h)$ $(h \in \mathfrak{h})$ on a vector $x u^{(k)}(\mathfrak{X})(x \in \mathfrak{g})$ is given by

$$
x u^{(k)}(\mathfrak{X}) S(h)=-\sum_{\lambda \in P_{+}^{(k)}} \sum_{i}\left(u_{\lambda}^{i} h\right) \otimes\left(x u_{\lambda, i}\right) .
$$

The $P$-grading on the space of coinvariants (3.8) of Theorem 3.6 is induced from the above $P$-grading.

In general, let $W=\oplus_{\nu \in P} W_{\nu}=\lim ^{j}$ be a $P$-graded vector space equipped with a filtration $0=F^{-1} \subset \vec{F}^{0} \subset F^{1} \subset \cdots$ by $P$-graded subspaces $F^{j}$. We define the character of the associated graded space by

$$
\operatorname{chgr} W=\sum_{j \in \mathbb{Z}_{\geq 0}, \nu \in P} \operatorname{dim}\left(F^{j} / F^{j-1}\right)_{\nu} q^{j} e^{\nu},
$$

where $e^{\nu}$ stands for the formal exponential symbol. When the $P$-grading is trivial, we use also the notation $\mathrm{ch}_{q}$.

The foregoing discussions lead us to consider three kinds of polynomials in $q, \mathcal{S}_{\lambda}\left(V_{1}, \cdots, V_{N}\right), \mathcal{K}_{\lambda}\left(V_{1}, \ldots, V_{N}\right)$ and $\mathcal{K}_{\lambda}^{(k)}\left(V_{1}, \ldots, V_{N}\right)$.

\section{Fusion $q$-supernomial coefficient}

$$
\operatorname{ch} \operatorname{gr} \mathcal{F}_{\mathcal{Z}}\left(V_{1}, \cdots, V_{N}\right)=\sum_{\lambda \in P} e^{\lambda} \mathcal{S}_{\lambda}\left(V_{1}, \cdots, V_{N}\right) .
$$

\section{Fusion Kostka polynomial/}

$$
\mathcal{K}_{\lambda}\left(V_{1}, \ldots, V_{N}\right)=\operatorname{ch} \operatorname{gr}\left(\mathcal{F}_{Z}\left(V_{1}, \cdots, V_{N}\right) / S\left(I_{\lambda}\right)\right),
$$

\section{Fusion restricted Kostka polynomial}

$$
\mathcal{K}_{\lambda}^{(k)}\left(V_{1}, \ldots, V_{N}\right)=\operatorname{ch} \operatorname{gr}\left(\mathcal{F}_{Z}\left(V_{1}, \cdots, V_{N}\right) / S\left(I_{\lambda}^{(k)}\right)\right) .
$$

The first two are simply related by an alternating sum over the Weyl group as

$$
\mathcal{K}_{\lambda}\left(V_{1}, \ldots, V_{N}\right)=\sum_{w \in W}(-1)^{l(w)} \mathcal{S}_{w \circ \lambda}\left(V_{1}, \ldots, V_{N}\right) .
$$

It is also clear that $\mathcal{K}_{\lambda}\left(V_{1}, \ldots, V_{N}\right)$ is obtained from $\mathcal{K}_{\lambda}^{(k)}\left(V_{1}, \ldots, V_{N}\right)$ in the limit $k \rightarrow \infty$.

We expect that, in the case when $V_{1}, \cdots, V_{N}$ are tensor powers of the fundamental representations discussed in [HKKOTY], [SW2], the 'fusionproduct' version defined above reduces to the corresponding object known in the literature. We expect also the following relation:

\footnotetext{
${ }^{2}$ The fusion Kostka polynomial defined in BKMW is different from ours.
} 


\section{Conjecture.}

$$
\mathcal{K}_{\lambda}^{(k)}\left(V_{1}, \ldots, V_{N}\right)=\sum_{\substack{w \in W_{\text {aff }} \\ w \circ \lambda \in P_{+}}}(-1)^{l(w)} q^{d_{w}(\lambda, k)} \mathcal{K}_{w \circ \lambda}\left(V_{1}, \ldots, V_{N}\right)
$$

The quantity $d_{w}(\lambda, k)$ is defined in (2.8). For the cases corresponding to Examples 2,3, (3.19) was put forward in [FU] as the 'main conjecture'.

\section{THE CASE $\mathfrak{g}=\mathfrak{s l}_{2}$}

In this section we restrict ourselves to the case $\mathfrak{g}=\mathfrak{s l}_{2}$ as mentioned in (A.6). In this special case, we shall identify the fusion-product version of Kostka/level-restricted Kostka polynomials with the ordinary ones and prove the identity (3.19). As before, we write $\pi_{l}$ for the $(l+1)$-dimensional irreducible representation and $[l]$ for the corresponding element of $\mathcal{V}^{(k)}$. We also write the left ideal (3.2) as $I_{l}^{(k)}$, and $S\left(I_{l}^{(k)}\right)$ as $\left\langle h[0]+l, e[0], e[1]^{k-l+1}\right\rangle$, where $x[i]=x \otimes t^{i}$.

4.1. level-restricted Kostka polynomials. First we recall a few basic facts about Kostka and level-restricted Kostka polynomials for $\mathfrak{s l}_{2}$, following the exposition of [SS]. We fix the notation as follows. Let $\mathbf{m}=\left(m_{1}, \cdots, m_{k}\right)$ be a $k$-tuple of non-negative integers, and let $0 \leq l \leq k$. Here and after, we use boldface letters to represent a vector. The transposition symbol is omitted. Set

$$
\begin{aligned}
& K_{l, \mathbf{m}}(q)=q^{\|\mathbf{m}\|} K_{\lambda R(\mathbf{m})}\left(q^{-1}\right), \\
& K_{l, \mathbf{m}}^{(k)}(q)=q^{\|\mathbf{m}\|} K_{\lambda R(\mathbf{m})}^{k}\left(q^{-1}\right) .
\end{aligned}
$$

Here the right hand sides stand for the Kostka and level-restricted Kostka polynomials in the notation of [SS],

$$
\begin{aligned}
& \lambda=\left(\frac{|\mathbf{m}|+l}{2}, \frac{|\mathbf{m}|-l}{2}\right), \\
& R(\mathbf{m})=\left(k^{m_{k}}, \cdots, 2^{m_{2}}, 1^{m_{1}}\right),
\end{aligned}
$$

and $|\mathbf{m}|=\sum_{i=1}^{k} i m_{i}, 2\|\mathbf{m}\|=\sum_{1 \leq i, j \leq k} \min (i, j) m_{i} m_{j}-|\mathbf{m}|$. Set further

$$
A_{a b}=\min (a, b), \quad v_{a}=\max (a-k+l, 0) .
$$

We use the $q$-binomial symbol

$$
\begin{aligned}
& {\left[\begin{array}{c}
\mathbf{m} \\
\mathbf{n}
\end{array}\right]=\prod_{i=1}^{k}\left[\begin{array}{c}
m_{i} \\
n_{i}
\end{array}\right],} \\
& {\left[\begin{array}{c}
m \\
n
\end{array}\right]= \begin{cases}\frac{[m] !}{[n] ![m-n] !} & (0 \leq n \leq m), \\
0 & \text { otherwise }\end{cases} }
\end{aligned}
$$

where $[n] !=\prod_{j=1}^{n}\left(\left(1-q^{j}\right) /(1-q)\right)$. We shall use the following facts. 
Fermionic formula:

$$
K_{l, \mathbf{m}}^{(k)}(q)=\sum_{\substack{\mathbf{s} \in \mathbb{Z}_{\geq 0}^{k} \\
2|\mathbf{s}|=|\mathbf{m}|-l}} q^{\mathbf{s} A \mathbf{s}+\mathbf{v s}}\left[\begin{array}{c}
A(\mathbf{m}-2 \mathbf{s})-\mathbf{v}+\mathbf{s} \\
\mathbf{s}
\end{array}\right] .
$$

\section{Alternating sum formula:}

$$
\begin{aligned}
K_{l, \mathbf{m}}^{(k)}(q)= & \sum_{i \geq 0} q^{(k+2) i^{2}+(l+1) i} K_{2(k+2) i+l, \mathbf{m}}(q) \\
& -\sum_{i>0} q^{(k+2) i^{2}-(l+1) i} K_{2(k+2) i-l-2, \mathbf{m}}(q) .
\end{aligned}
$$

Verlinde number: In the Verlinde algebra $\mathcal{V}^{(k)}$, we have

$$
[1]^{m_{1}} \cdots[k]^{m_{k}}=\sum_{l=0}^{k} K_{l, \mathbf{m}}^{(k)}(1)[l] .
$$

Eq. (4.2) and (4.3) are taken from Theorem 6.2 and eq. (6.8) in SS, respectively (after simplifications due to $n=2$ ). In HKKOTY, $K_{l, \mathbf{m}}^{(k)}(q)$ is defined as a generating sum over level $k$ restricted paths in affine crystals. With this definition, (4.4) follows from the fact that the structure constants of the Verlinde algebra are the same as the number of successive triples in a path satisfying the level-restriction conditions.

4.2. The goal. The fusion product is defined to be the associated graded vector space of the filtered tensor product

$$
V_{1} * \cdots * V_{N}(z)=\operatorname{gr} \mathcal{F}_{Z}\left(V_{1}, \cdots, V_{N}\right) .
$$

It gives a meaning to the 'limit' of the filtered tensor product where all points $z_{i}$ tend to 0 , as explained in Appendix. We consider the filtered tensor product and fusion product of cyclic modules $V_{i}=\pi_{l_{i}}$, choosing the highest weight vector as cyclic vector. Let $\mathbf{m}=\left(m_{1}, \cdots, m_{k}\right)$ and set

$$
V_{\mathbf{m}}=\overbrace{\pi_{k} * \cdots * \pi_{k}}^{m_{k}} * \cdots * \overbrace{\pi_{1} * \cdots * \pi_{1}}^{m_{1}}(z) .
$$

It is known (for $\mathfrak{g}=\mathfrak{s l}_{2}$ ) that (4.5) is independent of $z$ [FL, FF]. Our goal is to show the following.

Theorem 4.1. (i)Notations being as above, we have

$$
\operatorname{ch}_{q} V_{\mathbf{m}} /\left\langle h[0]+l, e[0], e[1]^{k-l+1}\right\rangle=K_{l, \mathbf{m}}^{(k)}(q) .
$$

(ii) The identity (3.19) is true in this case.

In the next subsection, we prove the inequality

$$
\operatorname{ch}_{q} V_{\mathbf{m}} /\left\langle h[0]+l, e[0], e[1]^{k-l+1}\right\rangle \leq K_{l, \mathbf{m}}^{(k)}(q) .
$$

Namely, each coefficient of powers in $q$ in the right hand side is greater than or equal to that in the left hand side.

Assuming (4.6), let us prove Theorem 4.1. 
Proof of Theorem 4.1. From Corollary 3.7 and (A.3), we have for any $V_{i}=\pi_{l_{i}}$ that

$$
\begin{aligned}
\left(\left[l_{1}\right] \cdots\left[l_{N}\right]:[l]\right)_{k}= & \operatorname{dim} \mathcal{F}_{z}\left(V_{1}, \cdots, V_{N}\right) /\left\langle h[0]+l, e[0], e[1]^{k-l+1}\right\rangle \\
& \leq \operatorname{dim} V_{1} * \cdots * V_{N} /\left\langle h[0]+l, e[0], e[1]^{k-l+1}\right\rangle .
\end{aligned}
$$

Combining this with (4.4), we obtain

$$
K_{l, \mathbf{m}}^{(k)}(1) \leq \operatorname{dim} V_{\mathbf{m}} /\left\langle h[0]+l, e[0], e[1]^{k-l+1}\right\rangle .
$$

Therefore, it follows from (4.6) that equality takes place in all intermediate steps. In particular, assertion (i) is implied by the equality in (4.6). Assertion (ii) is then an immediate consequence of the known alternating sum formula (4.3) for level-restricted Kostka polynomials.

4.3. Gordon filtration. It remains to prove the inequality (4.6). For this purpose we invoke the 'functional' description of the dual space [FS].

It was shown in [FF] that, as graded vector space, the dual of $V_{\mathbf{m}}$ is isomorphic to the space of all symmetric polynomials $f\left(x_{1}, \cdots, x_{s}\right)(s \geq 0)$ satisfying the condition

$$
\begin{gathered}
\operatorname{deg}_{x} f(\overbrace{x, \cdots, x}^{a}, x_{a+1}, \cdots, x_{s}) \leq M_{a}-a \\
\text { for any } a=1 \cdots, s,
\end{gathered}
$$

where

$$
M_{a}=\sum_{i=1}^{k} \min (a, i) m_{i} .
$$

Therefore the dual to the quotient space of (4.5) by $\left\langle h[0]+l, e[0], e[1]^{k-l+1}\right\rangle$ is realized as follows.

Lemma 4.2. As a graded vector space, $\left(V_{\mathbf{m}} /\left\langle h[0]+l, e[0], e[1]^{k-l+1}\right\rangle\right)^{*}$ is isomorphic to the space $\mathcal{F}$ of all symmetric polynomials satisfying (4.7) as well as the conditions

(i) The number of variables is $s=\frac{1}{2}(|\mathbf{m}|-l)$,

(ii) $f\left(0, x_{2}, \cdots, x_{s}\right)=0$,

(iii) If $s \geq k-l+1$, then setting $f\left(x_{1}, \cdots, x_{s}\right)=\left(\prod_{i=1}^{s} x_{i}\right) g\left(x_{1}, \cdots, x_{s}\right)$ we have

$$
g(\overbrace{0, \cdots, 0}^{k-l+1}, x_{k-l+2}, \cdots, x_{s})=0 .
$$

Lemma 4.3. Assume that $s \geq k+1$. For any $f \in \mathcal{F}$ we have

$$
f(\overbrace{x, \cdots, x}^{a}, x_{a+1}, \cdots, x_{s})=0 \quad(\text { if } a \geq k+1) .
$$


Proof. Let $g\left(x_{1}, \cdots, x_{s}\right)$ be as in Lemma 4.2 (iii). From (4.8), we find

$$
\begin{array}{r}
\left.\left(\frac{\partial}{\partial x}\right)^{j} g(\overbrace{x, \cdots, x}^{a}, x_{a+1}, \cdots, x_{s})\right|_{x=0}=0 \\
(0 \leq j \leq l, a \geq k+1) .
\end{array}
$$

Namely we have

$$
g(\overbrace{x, \cdots, x}^{a}, x_{a+1}, \cdots, x_{s})=O\left(x^{l+1}\right) .
$$

We prove the assertion by descending induction on $a$. First let $a=s$. Since $s \geq k+1$, we have $M_{a}=|\mathbf{m}|$. Then (4.7) and Lemma 4.2 (i) imply $\operatorname{deg}_{x} g(x, \cdots, x) \leq|\mathbf{m}|-2 s=l$. Hence we have $g(x, \cdots, x)=0$ by (4.10). Suppose the assertion is true for $a+1(a \geq k+1)$. Then $g\left(x_{1}, \cdots, x_{s}\right)$ can be written as

$$
g(\overbrace{x, \cdots, x}^{a}, x_{a+1}, \cdots, x_{s})=\prod_{i=a+1}^{s}\left(x-x_{i}\right)^{2} h\left(x ; x_{a+1}, \cdots, x_{s}\right) .
$$

From (4.7) we find $\operatorname{deg}_{x} h \leq l$. Therefore (4.10) implies $h=0$, and the proof is over.

In order to estimate the character of $\mathcal{F}$, we apply the standard technique of Gordon filtration [FS. Let $\lambda$ be a partition of width $\lambda_{1} \leq k$, and write $\lambda=\left(k^{s_{k}} \cdots 2^{s_{2}} 1^{s_{1}}\right)$. For a symmetric polynomial $f$ we define

$$
\begin{aligned}
& \varphi_{\lambda}(f)\left(x_{1}^{(1)}, \cdots, x_{s_{1}}^{(1)} ; \cdots ; x_{1}^{(k)}, \cdots, x_{s_{k}}^{(k)}\right) \\
& =f(x_{1}^{(1)}, \cdots, x_{s_{1}}^{(1)}, \cdots, \overbrace{x_{1}^{(k)}, \cdots, x_{1}^{(k)}}^{k}, \cdots, \overbrace{x_{s_{k}}^{(k)}, \cdots, x_{s_{k}}^{(k)}}^{k}),
\end{aligned}
$$

where each variable $x_{i}^{(a)}$ appears $a$ times in the right hand side. Introduce the lexicographic ordering $\mu>\lambda$ by $\mu_{1}=\lambda_{1}, \cdots, \mu_{i-1}=\lambda_{i-1}$ and $\mu_{i}>\lambda_{i}$ for some $i$, and set

$$
\mathcal{F}_{\lambda}=\bigcap_{\mu>\lambda} \operatorname{Ker} \varphi_{\mu}
$$

Notice that $\mathcal{F}_{(k)}=\mathcal{F}$ by Lemma 4.3. The family of subspaces $\left\{\mathcal{F}_{\lambda}\right\}_{\lambda}$ defines a filtration of $\mathcal{F}$, whose associated graded space has the $\lambda$-th component isomorphic to $\varphi_{\lambda}\left(\mathcal{F}_{\lambda}\right)$. An element of the latter has the form

$$
\begin{aligned}
& \left(\prod_{\substack{1 \leq i \leq s_{a} \\
1 \leq a \leq k}} x_{i}^{(a)}\right)^{a+(a-k+l)_{+}} \prod_{\substack{1 \leq i \leq j \leq s_{a} \\
1 \leq a \leq k}}\left(x_{i}^{(a)}-x_{j}^{(a)}\right)^{2 a} \\
& \times \prod_{\substack{1 \leq i \leq s_{a}, 1 \leq j \leq s_{b} \\
1 \leq a<b \leq k}}\left(x_{i}^{(a)}-x_{j}^{(b)}\right)^{2 \min (a, b)} \cdot h,
\end{aligned}
$$

with a partially symmetric polynomial

$$
h \in \mathbb{C}\left[x_{1}^{(1)}, \cdots, x_{s_{1}}^{(1)}, \cdots, x_{1}^{(k)}, \cdots, x_{s_{k}}^{(k)}\right]^{\mathfrak{S}_{s_{1}} \times \cdots \times \mathfrak{S}_{s_{k}}},
$$


$\mathfrak{S}_{s}$ denoting the symmetric group on $s$ letters. We have the relations for the degree

$$
\begin{aligned}
& \operatorname{deg}_{x_{i}^{(a)}} h \leq(A(\mathbf{m}-2 \mathbf{s})-\mathbf{v})_{a}, \\
& \text { tot. } \operatorname{deg} f=\text { tot. } \operatorname{deg} h+\mathbf{s} A \mathbf{s}+\mathbf{v s},
\end{aligned}
$$

where we used the vector notation (4.1), and tot. deg signifies the total degree. We thus obtain for the character $\operatorname{ch}_{q}$ gr $\mathcal{F}$ an upper bound

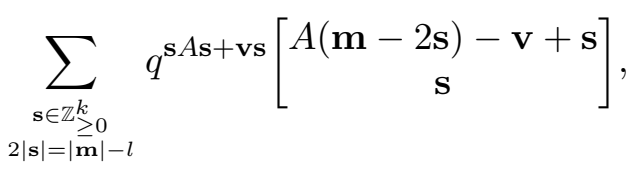

which coincides with the fermionic form of the level-restricted Kostka polynomial (4.2). This completes the proof of (4.6).

\section{Appendix A. Degeneration of Coinvariants and fusion product}

Here we give a motivation for the notion of fusion product introduced in [FL and used in Section 4.

In this paper we have considered fusion of congruence right ideals of $U(\mathfrak{g}[t])$ attached to distinct points. They are parameterized by the configuration space of complex points. In general, it is not clear what is the analog of fusion when points collide, but we can extend the definition to the boundary of the configuration space $\mathbf{C}^{N} \backslash \bigcup \Delta_{[a, b]}$ (see $[$ FM] ) in the following way.

For $a \in \mathbb{C} \backslash\{0\}$ we define an automorphism $l_{a}: \mathfrak{g}[t] \rightarrow \mathfrak{g}[t]$ multiplying the variable $t$ by $a^{-1}$. Clearly we have

$$
l_{a}\left(X_{1} \circledast \ldots \circledast X_{N}(z)\right)=X_{1} \circledast \ldots \circledast X_{N}(a z) .
$$

These spaces form a family over $\mathbb{C} \backslash\{0\}$, and we want to extend it to the origin.

Definition 3. For a subspace $W^{\prime}$ of a graded vector space $W=\oplus_{j \geq 0} W_{j}$, we denote by $W^{\text {top }}$ the graded subspace of $W$ spanned by homogeneous elements $w \in W_{r}$, such that for some $w_{j} \in W_{j}(0 \leq j \leq r)$ we have $w=w_{r}$ and $w_{r}+w_{r-1}+\cdots+w_{0} \in W^{\prime}$.

Note that $\mathfrak{g}[t]$ and therefore $U(\mathfrak{g}[t])$ has a natural grading by degree of $t$. Hence, for any subspace $X \subset U(\mathfrak{g}[t])$ we can consider the subspace $X^{\text {top }} \subset$ $U(\mathfrak{g}[t])$. In particular, the space $\left(X_{1} \circledast \ldots \circledast X_{N}(z)\right)^{\text {top }}$ can be thought of as the limit of $X_{1} \circledast \ldots \circledast X_{N}(a Z)$ when the parameter $a$ tends to 0 .

More generally, combining the operation of $\circledast$ and taking $(\ldots)^{\text {top }}$ in various ways, one can extend the definition of fusion to the compactification 
$\mathbb{C}[N]$ of the configuration space in the sense of $[\mathrm{FM}]$. For example, corresponding to Figure 4 in [FM], we have the following fusion of right ideals:

$$
\begin{aligned}
& Y_{1}=\left(X_{1} \circledast X_{3} \circledast X_{8}\left(z_{1}, z_{3}, z_{8}\right)\right)^{\mathrm{top}}, \\
& Y_{2}=\left(X_{4} \circledast X_{6}\left(z_{4}, z_{6}\right)\right)^{\mathrm{top}}, \\
& Y_{3}=\left(X_{2} \circledast X_{5}\left(z_{2}, z_{5}\right)\right)^{\mathrm{top}}, \\
& Y_{4}=\left(Y_{1} \circledast Y_{2}\left(z_{138}, z_{46}\right)\right)^{\mathrm{top}}, \\
& Y_{5}=\left(Y_{4} \circledast Y_{3} \circledast X_{7}\left(z_{13468}, z_{25}, z_{7}\right)\right)^{\mathrm{top}} .
\end{aligned}
$$

Question 1. When is the ideal $\left(X_{1} \circledast \ldots \circledast X_{N}(z)\right)^{\text {top }}$ independent of z? More generally, when do the extended fusion right ideals depend only on the image of the point in $\mathbb{C}[N]$ under the natural map $\mathbb{C}[N] \rightarrow \mathbb{C}^{N}$ ?

In the following situation the top part of the right ideal is given rather explicitly.

Proposition A.1. Let $\mathfrak{b} \subset \mathfrak{g}[t]$ be a Lie subalgebra. Then, we have

$$
\mathfrak{b}^{\text {top }} U(\mathfrak{g}[t])=(\mathfrak{b} U(\mathfrak{g}[t]))^{\text {top }} .
$$

Proof. Let $\mathbb{C}[t]_{\leq d}$ be the space of polynomials of degree less than or equal to $d$. Set $\mathfrak{b}_{\leq d}=\mathfrak{b} \cap \mathbb{C}[t]_{\leq d}$. One can construct a basis of $\mathfrak{b},\left\{B_{i}\right\}$, in such a way that for each $d$ the subset $\left\{B_{i}\right\} \cap \mathfrak{b}_{\leq d}$ constitutes a basis of $\mathfrak{b}_{\leq d}$. Then, $\left\{B_{i}^{\text {top }}\right\}$ is a basis of $\mathfrak{b}^{\text {top }}$. One can construct a homogeneous basis of $\mathfrak{g}[t]$ consisting of $\left\{B_{i}^{\text {top }}\right\}$ and new members $\left\{A_{i}\right\}$. Since $\mathfrak{b}$ is a Lie subalgebra, by Lemma 2.2 $B_{i_{1}} \cdots B_{i_{m}} A_{j_{1}} \cdots A_{j_{n}}$ where $m \geq 1, i_{1} \leq \cdots \leq i_{m}$ and $n \geq 0, j_{1} \leq \cdots \leq j_{n}$ constitute a basis of the right ideal $\mathfrak{b} U(\mathfrak{g}[t])$. Note that

$$
\left(B_{i_{1}} \cdots B_{i_{m}} A_{j_{1}} \cdots A_{j_{n}}\right)^{\text {top }}=B_{i_{1}}^{\text {top }} \cdots B_{i_{m}}^{\text {top }} A_{j_{1}} \cdots A_{j_{n}}
$$

constitute a basis of $(\mathfrak{b} U(\mathfrak{g}[t]))^{\text {top }}$. Since $\mathfrak{b}^{\text {top }}$ is also a Lie subalgebra, these elements constitute a basis of $\mathfrak{b}^{\text {top }} U(\mathfrak{g}[t])$. Therefore, we have the equality of the right ideals (A.1).

In particular, we have a positive answer to Question 1 for $X_{i}=B_{n_{i}}$, since

$$
\left(B_{n_{1}} \circledast \ldots \circledast B_{n_{N}}\right)^{\text {top }}=B_{n_{1}+\cdots+n_{N}} .
$$

Now let us proceed to coinvariants. By the definition, there is a canonical isomorphism of graded vector space

$$
\operatorname{gr}\left(W / W^{\prime}\right) \simeq W / W^{\prime \text { top }} .
$$

On the other hand, if $X$ is a right ideal of a graded algebra $U$ acting on $W$, the dimension of the space of coinvariants may jump in general.

$$
\operatorname{dim} W / X W \leq \operatorname{dim} W / X^{\text {top }} W .
$$

Proposition A.2. There is a surjective map

$$
L_{\lambda}^{(k)}(\infty) /\left(X_{1} \circledast \ldots \circledast X_{N}(z)\right)^{\text {top }} \rightarrow \operatorname{gr} L_{\lambda}^{(k)}(\infty) /\left(X_{1} \circledast \ldots \circledast X_{N}(z)\right)
$$


Question 2. When is the map (A.4) an isomorphism? More generally, when is the dimension of coinvariants independent of the point in $\mathbb{C}[N]$ ?

The answer to this question for $X_{i}=B_{n_{i}}$ is positive for $\mathfrak{g}=s l_{2}$ (see [FKLMM1]). On the other hand, if $\mathfrak{g}=E_{8}$, it is negative even for $X_{i}=B_{1}$ with $k=1$. This is because for level 1 the only integrable representation is the vacuum representation, i.e., the highest weight is 0 . Therefore, the right hand side is always one-dimensional, while the left hand side becomes infinite in the limit $N \rightarrow \infty$.

Similar considerations for the filtered tensor product of modules can be found in [FL]. Recall that the associated graded vector space of the filtered tensor product

$$
V_{1} * \cdots * V_{N}(Z)=\operatorname{gr} \mathcal{F}_{Z}\left(V_{1}, \cdots, V_{N}\right)
$$

is called the fusion product. It gives a meaning to the similar limit of the filtered tensor product where all points $z_{i}$ tend to 0 . In FL it was conjectured that, for finite-dimensional cyclic modules over a simple Lie algebra $\mathfrak{g}$, the fusion product $V_{1} * \cdots * V_{N}(Z)$ is independent of the choice of $z$. This conjecture was proved in [FL] in the simplest case where

$$
\begin{aligned}
& \mathfrak{g}=\mathfrak{s l}_{2}, \quad V_{i}: \text { irreducible module } \\
& \text { with highest weight vector as cyclic vector, }
\end{aligned}
$$

and in $[\mathrm{FF}$ by a different method. Indeed, the fusion product can also be extended to $\mathbb{C}[N]$ and it can be proved by the method of $[\mathrm{FF}]$ that in this case it depends only on the image in $\mathbb{C}^{N}$.

An advantage of this definition is that we can split the map (A.4) into two.

Theorem A.3. $\quad$ (i) The map (A.4) is a composition of the following surjective maps

$$
\begin{aligned}
& \text { (A.7) } L_{\lambda}^{(k)}(\infty) /\left(X_{1} \circledast \cdots \circledast X_{N}(\mathbb{Z})\right)^{\text {top }} \rightarrow \pi^{(k)}\left(\mathfrak{X}_{1}\right) * \cdots * \pi^{(k)}\left(\mathfrak{X}_{N}\right) / S\left(I_{\lambda}^{(k)}\right) \\
& \text { (A.8) } \pi^{(k)}\left(\mathfrak{X}_{1}\right) * \cdots * \pi^{(k)}\left(\mathfrak{X}_{N}\right) / S\left(I_{\lambda}^{(k)}\right) \rightarrow \operatorname{gr} L_{\lambda}^{(k)}(\infty) /\left(X_{1} \circledast \cdots \circledast X_{N}(Z)\right)
\end{aligned}
$$

(ii) Assume that $\mathfrak{X}_{i} \supset \mathfrak{I}^{(k)}$. Then the map (A.7) is an isomorphism.

Proof. To prove (i) we just follow the proof of Theorem 3.6.

Let $\tilde{\mathfrak{X}}_{i}=\mathfrak{X}_{i}+\mathfrak{I}^{(k)}$. As usual, set $X_{i}=B_{1}+S\left(\mathfrak{X}_{i}\right), \tilde{X}_{i}=B_{1}+S\left(\tilde{\mathfrak{X}}_{i}\right)$. Then the natural projection

$$
L_{\lambda}^{(k)}(\infty) /\left(X_{1} \circledast \cdots \circledast X_{N}(Z)\right) \rightarrow L_{\lambda}^{(k)}(\infty) /\left(\tilde{X}_{1} \circledast \cdots \circledast \tilde{X}_{N}(z)\right)
$$

is an isomorphism.

So we can split the map (A.4) into composition of the projection

$$
L_{\lambda}^{(k)}(\infty) /\left(X_{1} \circledast \cdots \circledast X_{N}(Z)\right)^{\text {top }} \rightarrow L_{\lambda}^{(k)}(\infty) /\left(\tilde{X}_{1} \circledast \cdots \circledast \tilde{X}_{N}(Z)\right)^{\text {top }}
$$


and the map (A.4) for $\tilde{X}_{i}$ :

$$
L_{\lambda}^{(k)}(\infty) /\left(\tilde{X}_{1} \circledast \cdots \circledast \tilde{X}_{N}(Z)\right)^{\text {top }} \rightarrow \operatorname{gr} L_{\lambda}^{(k)}(\infty) /\left(\tilde{X}_{1} \circledast \cdots \circledast \tilde{X}_{N}(Z)\right) .
$$

To prove (i) it remains to identify $L_{\lambda}^{(k)}(\infty) /\left(\tilde{X}_{1} \circledast \cdots \circledast \tilde{X}_{N}(Z)\right)^{\text {top }}$ with the space $\pi^{(k)}\left(\mathfrak{X}_{1}\right) * \cdots * \pi^{(k)}\left(\mathfrak{X}_{N}\right) / S\left(I_{\lambda}^{(k)}\right)$.

We have

$$
\begin{aligned}
& L_{\lambda}^{(k)}(\infty) /\left(\tilde{X}_{1} \circledast \cdots \circledast \tilde{X}_{N}(Z)\right)^{\text {top }} \\
\simeq \quad & U(\mathfrak{g}[t]) /\left(I_{\lambda}^{(k)}+U(\mathfrak{g}[t]) \operatorname{Ann} \pi_{\lambda}\right) /\left(\tilde{X}_{1} \circledast \cdots \circledast \tilde{X}_{N}(Z)\right)^{\text {top }}
\end{aligned}
$$

(by Proposition 3.2)

$$
\simeq \quad U(\mathfrak{g}[t]) /\left(\tilde{X}_{1} \circledast \cdots \circledast \tilde{X}_{N}(z)\right)^{\mathrm{top}} /\left(I_{\lambda}^{(k)}+U(\mathfrak{g}[t]) \operatorname{Ann} \pi_{\lambda}\right)
$$

(as a bi-quotient by the left and right ideals)

$$
\simeq \quad \operatorname{gr}\left(U(\mathfrak{g}[t]) / \tilde{X}_{1} \circledast \cdots \circledast \tilde{X}_{N}(z)\right) /\left(I_{\lambda}^{(k)}+U(\mathfrak{g}[t]) \operatorname{Ann} \pi_{\lambda}\right)
$$

(by A.2 and using that $I_{\lambda}^{(k)}$ is homogeneous)

$$
\simeq \quad \pi^{(k)}\left(\mathfrak{X}_{1}\right) * \cdots * \pi^{(k)}\left(\mathfrak{X}_{N}\right) /\left(S\left(I_{\lambda}^{(k)}\right)+\operatorname{Ann} \pi_{\lambda^{*}} U(\mathfrak{g}[t])\right)
$$

(by Proposition 3.5 and by $($ A.5 $)$ )

$$
\simeq \pi^{(k)}\left(\mathfrak{X}_{1}\right) * \cdots * \pi^{(k)}\left(\mathfrak{X}_{N}\right) / S\left(I_{\lambda}^{(k)}\right) .
$$

(by repeating the last part of the proof of Theorem 3.6)

To prove (ii) just note that $\mathfrak{X}_{i} \supset \mathfrak{I}^{(k)}$ implies $X_{i}=\tilde{X}_{i}$, so the first map is an isomorphism.

Let us illustrate how it works for the case of conformal blocks. For simplicity suppose $\mathfrak{g}=\mathfrak{s l}_{2}$.

For this purpose we need ideals $\mathfrak{X} \subset U(\mathfrak{g})$ such that $\pi^{(k)}(\mathfrak{X})=\pi_{l}$. Actually, we can choose them in different ways. Following (2.19) and (2.20) set

$$
\begin{gathered}
\mathfrak{X}_{l}=U\left(\mathfrak{s l}_{2}\right) e+U\left(\mathfrak{s l}_{2}\right)(h-l), \\
\mathfrak{X}_{l}^{\prime}=U\left(\mathfrak{s l}_{2}\right) e+U\left(\mathfrak{s l}_{2}\right)(h-l)+U\left(\mathfrak{s l}_{2}\right) f^{l+1} .
\end{gathered}
$$

Then $\mathfrak{X}_{l}^{\prime} \supset \mathfrak{I}^{(k)}$ because $\mathfrak{X}_{l}^{\prime}$ is the annihilating ideal of the highest weight vector in $\pi_{l}$ where $0 \leq l \leq k$. Therefore, by Theorem A.3 the map (A.7) is an isomorphism. In Section 4 we prove that the map (A.8) is an isomorphism, so the composition map (A.4) is an isomorphism, i.e., the answer to the Question 2 is positive in this case.

But this is not so for $\mathfrak{X}_{l}$. Namely, let us show that the map (A.7) can not always be an isomorphism.

Let $X_{i}=S\left(\mathfrak{X}_{l_{i}}\right)+B_{1}$. In a way similar to the proof of Proposition A.1, one can show that $X_{1} \circledast \cdots \circledast X_{N}(Z)$ is generated by $e \otimes t^{j}$ and $h \otimes t^{j}-\sum_{i=1}^{N} l_{i} z_{i}^{j}$ $(j \geq 0)$. Hence $\left(X_{1} \circledast \cdots \circledast X_{N}(Z)\right)^{\text {top }}$ is generated by $e \otimes t^{j}(j \geq 0)$, $h \otimes t^{j}(j>0)$ and $h-\sum_{i=1}^{N} l_{i}$. This means that the left hand side of (A.7) 
depends only on $\sum_{i=1}^{N} l_{i}$, but it is easy to see (e.g. by taking $k \rightarrow \infty$ ) that the dimension of the right hand side depends on each $l_{i}$.

Acknowledgments. This work is partially supported by the Grant-in-Aid for Scientific Research (A1) no.13304010 and (B2) no.14340040, Japan Society for the Promotion of Science. BF is partially supported by grants RFBR 02-01-01015 and INTAS-00-00055. The work of SL is partially supported by the grant RFBR-01-01-00546. The last stage of this work was carried out while the authors were visiting Mathematical Sciences Research Institute, Berkeley, March 2002.

\section{REFERENCES}

[BKMW] L. Begin, A.N. Kirillov, P. Mathieu and M.A. Walton, Berenstein-Zelevinski triangles, elementary coupling and fusion rules, Lett. Math. Phys., 28 (1993), 257-268.

$[\mathrm{FF}] \quad$ B. L. Feigin and E. Feigin, $q$-characters of the tensor products in $\mathfrak{s l}_{2}$-case, math.QA/0201111 (2002).

$[\mathrm{FFu}] \quad$ B. L. Feigin and D. B. Fuchs, Cohomology of some nilpotent subalgebras of the Virasoro and Kac-Moody algebras, Geometry and Physics, Essays in honor of I.M.Gelfand on the occasion of his 75th birthday, eds. S.Gindikin and I.M.Singer, 209-235, North Holland, (1991)

[FKLMM1] B. Feigin, R. Kedem, S. Loktev, T. Miwa and E. Mukhin, Combinatorics of $\widehat{\mathfrak{s l}}_{2}$ spaces of coinvariants, Transformation Groups 6 (2001) No.1 25-52.

[FKLMM2] B. Feigin, R. Kedem, S. Loktev, T. Miwa and E. Mukhin, Combinatorics of the $\widehat{\mathfrak{s l}}_{2}$ spaces of Coinvariants: Loop Heisenberg modules and recursion, math.QA/0009198

[FKLMM3] B. Feigin, R. Kedem, S. Loktev, T. Miwa and E. Mukhin, Combinatorics of the $\widehat{\mathfrak{s l}}_{2}$ spaces of coinvariants: Dual functional realization and recursion, math.QA/0012190

[FL] B. L. Feigin and S. Loktev, On generalized Kostka polynomials and quantum Verlinde rule, math.QA/9812093, Amer. Math. Sci. Transl. 194 (1999) 6179 .

[FM] W. Fulton and P. MacPherson. A compactfication of configuration spaces, Ann. Math. 139 (1994) 183-225.

[Fo] H. O. Foulkes, A survey of some combinatorial aspects of symmetric functions, in Permutations, Gauthier-Villars (1974), Paris.

[FS] B. L. Feigin and A. V. Stoyanovsky, Functional models for representations of current algebras and semi-imfinite Schubert cells, Funct. Anal. and Its Appl. 28 (1993) 55-72.

[HKKOTY] G. Hatayama, A. N. Kirillov, A. Kuniba, M. Okado, T. Takagi and Y. Yamada, Character formulae of $\widehat{s l}_{n}$-modules and inhomogeneous paths, math.QA/9802085, Nucl. Phys. B 536 (1999) 575-616.

[KKMM] R. Kedem, T. R. Klassen, B. M. McCoy and E. Melzer, Fermionic sum representations for conformal field theory characters, Phys. Lett. B 307 (1993) 68-76.

[KM] R. Kedem and B. M. McCoy, Construction of modular branching functions from Bethe's equations in the 3-state potts chain, J. Stat. Phys. 71 (1993) 875-901.

[KR] A. N. Kirillov and N. Yu. Reshetikhin, The Bethe Ansatz and the combinatorics of Young tableaux, J. Soviet Math. 41 (1988) 925-955. 
[KS] A. N. Kirillov and M. Shimozono, A generalization of the Kostka-Foulkes polynomials, math.QA/9804039, J. Algebraic Combin. 15 (2002) 27-69.

[KSS] A. N. Kirillov, M. Shimozono and A. Schilling, A bijection between Littlewood-Richardson tableaux and rigged configurations. (Preprint math.CO/9901037).

[LS] A. Lascoux and M.P. Schützenberger, Sur une conjecture de H. O. Foulkes, C. R. Acad. Sci. Paris Ser. A-B 286 (1978) A323-A324.

[Mac] I. G.Macdonald, Symmetric functions and Hall polynomials, 2nd ed., Oxford University Press, New York, 1995.

$[\mathrm{NY}] \quad$ A. Nakayashiki and Y. Yamada, Kostka polynomials and energy functions in solvable lattice models, Selecta Math. 3 (1997) 547-599.

[SS] A. Schilling and M. Shimozono, Fermionic formulas for level-restricted generalized Kostka polynomials and coset branching functions, math.QA/0001114, Commun. Math. Phys. 220 (2001) 105-164,

[SW1] A. Schilling and O. Warnaar, Supernomial coefficients, polynomial identities and $q$-series, Ramanujan J. 2 (1998) 459-494.

[SW2] A. Schilling and S. O. Warnaar, Inhomogeneous lattice paths, generalized Kostka polynomials and $A_{n-1}$ supernomials, math.QA/9802111, Commun. Math. Phys. 202 (1999) 359-401.

[TUY] A. Tsuchiya, K. Ueno and Y. Yamada, Conformal field theory on the universal family of stable curves with gauge symmetries, Adv. Stud. Pure Math. 19 (1989) 459-566.

BF: Landau institute for Theoretical Physics, Chernogolovka, 142432, RUSSIA

E-mail address: feigin@feigin.mccme.ru

MJ: Graduate School of Mathematical Sciences, The University of Tokyo, TOKYO 153-8914, JAPAN

E-mail address: jimbomic@ms.u-tokyo.ac.jp

RK: Department of Mathematics, University of Illinois, 1409 W. Green St. URBANA, IL 61801, USA

E-mail address: rinat@math.uiuc.edu

SL: Institute for Theoretical and Experimental Physics, B. CheremushrinSKAJA, 25, MOSCOW 117259, Russia

Independent University of Moscow, B. Vlasievsky per, 11, Moscow 121002, RUSSIA

E-mail address: loktev@mccme.ru

TM: Division of Mathematics, Graduate School of Science, Kyoto UniverSITY, КYОTO 606-8502 JAPAN

E-mail address: tetsuji@kusm.kyoto-u.ac.jp 